\title{
NK cell spatial dynamics and IgA responses in gut- associated lymphoid tissues during SIV infections
}

Philippe Rascle

Institut Pasteur https://orcid.org/0000-0001-8860-3938

Cyril PLANCHAIS

Institut Pasteur

Beatrice JACQUELIN

Institut Pasteur

Marie LAZZERINI

Institut Pasteur

Vanessa CONTRERAS

Université Paris

Caroline PASSAES

Institut Pasteur

Asier Saez-Cirion

Institut Pasteur https://orcid.org/0000-0003-2406-7536

Hugo MOUQUET

Institut Pasteur

Nicolas HUOT

Institut Pasteur

Michaela MULLER-TRUTWIN ( $\nabla$ michaela.muller-trutwin@pasteur.fr)

Institut Pasteur

Article

Keywords: HIV, SIV, NK cells

Posted Date: July 6th, 2021

DOI: https://doi.org/10.21203/rs.3.rs-643541/v1

License: (1) This work is licensed under a Creative Commons Attribution 4.0 International License.

Read Full License 
Version of Record: A version of this preprint was published at Communications Biology on July 7th, 2022. See the published version at https://doi.org/10.1038/s42003-022-03619-y. 


\section{NK cell spatial dynamics and IgA responses in gut-associated lymphoid tissues during SIV infections}

Philippe RASCLE ${ }^{1,2}$, Cyril PLANCHAIS ${ }^{3,4}$, Béatrice JACQUELIN ${ }^{1}$, Marie LAZZERINI ${ }^{1}$, Vanessa CONTRERAS ${ }^{5}$, Caroline PASSAES ${ }^{1}$, Asier SAEZ-CIRION ${ }^{1}$, Hugo MOUQUET ${ }^{3,4}$, Nicolas HUOT $^{1}$ and Michaela MÜLLER-TRUTWIN ${ }^{1, *}$.

${ }^{1}$ Institut Pasteur, Unité HIV Inflammation and Persistance, Paris, France;

${ }^{2}$ Université Paris Diderot, Sorbonne Paris Cité, Paris, France,

${ }^{3}$ Institut Pasteur, Laboratory of Humoral Immunology, Paris, France;

${ }^{4}$ INSERM U1222, Paris, France ;

${ }^{5}$ CEA, Université Paris Sud 11, INSERM U1184, Immunology of Viral Infections and Autoimmune Diseases, IDMIT, IBFJ, Fontenay-aux-Roses, France.

Keywords :

${ }^{*}$ Corresponding author :

Michaela Müller-Trutwin

Institut Pasteur

28, rue du Dr Roux

75724 Paris Cedex 15, France

mmuller@pasteur.fr (Orcid ID: 0000-0002-3854-2396) 


\begin{abstract}
HIV infection induces tissue damage including lymph node (LN) fibrosis and intestinal epithelial barrier disruption leading to bacterial translocation and systemic inflammation. Natural hosts of SIV, such as African Green Monkeys (AGM), do not display tissue damage despite high viral load in blood and intestinal mucosa. AGM mount a NK cell-mediated control of SIVagm replication in peripheral LN. We analyzed if NK cells also control SIVagm in mesenteric (mes) LN and if this has an impact on gut humoral responses, in particular on the production of IgA known for their anti-inflammatory role in the gut. We show that NK cells migrate into and control viral replication in B cell follicles (BCF) of mesLN during SIVagm infection. Ig $\mathrm{A}^{+}$memory $\mathrm{B}$ cells were increased in mesLN during SIVagm infection in contrast to SIVmac infection and correlated positively with CXCR5 ${ }^{+} \mathrm{NK}$ cell levels. Total IgA levels in gut remained normal during SIVagm infection, while strongly decreased in intestinal of chronically SIVmac-infected macaques. Systemic IgA titers correlated negatively with SCD14 levels in SIVmac infection. Our data suggest an indirect impact of NK cell-mediated viral control in mesLN during SIVagm infection on preserved BCF function and IgA production in intestinal tissues.
\end{abstract}




\section{INTRODUCTION}

The immune system evolved to limit the negative effects exerted by pathogens on host homeostasis. Infections generally result in tissue damage, especially if replication of the pathogen is not controlled ${ }^{1}$. Tissue damage can be triggered directly by pathogens or indirectly by host responses to the infection. Disease tolerance is a defense strategy against tissue damage induced by chronic infection that sustains host homeostasis, without exerting a direct negative impact on pathogens ${ }^{2}$. In people living with HIV (PLWH), anti-retroviral treatment (ART) has transformed a deadly disease into a manageable chronic infection. However, a residual chronic inflammation persists in most PLWH, in particular in individuals who started ART only after several years of infection in chronic infection, which still is the most frequent case. This chronic inflammation in PLWH under effective ART is associated with the risk of non-AIDS morbidities and mortality ${ }^{3,4,5}$. One factor that might largely contribute to the persistent inflammation is the disruption of the intestinal barrier and subsequent bacterial translocation in $\mathrm{PLWH}^{6,7}$. ART treatment is not able to eliminate the virus, which hides throughout the body in reservoirs. The largest HIV reservoir resides in the intestine $^{8,9}$. Residual viral replication can also be observed in follicular helper CD4+ $T\left(T_{F H}\right)$ cells of $B$ cell follicles (BCF) of lymph nodes (LN) during chronic infection in long-term treated $\mathrm{PLWH}^{10}$.

Natural hosts of SIV, such as African Green Monkeys (AGMs), do not display chronic inflammation despite stable high viremia during SIV infection ${ }^{11,6}$. Interestingly, SIV-infected AGMs generally do not show any major tissue damage. Thus, LN do not display fibrosis and the follicular dendritic cell (FDC) network is maintained throughout infection in contrast to $\mathrm{PLWH}^{12}$. The intestinal epithelial barrier also remains intact ${ }^{13,14}$ and no microbial translocation occurs ${ }^{14,15,16}$. The maintenance of normal $L N$ architecture can be explained by a rapid and strong viral control in this site ${ }^{17}$. Indeed, AGM mount a tissue-specific viral control in secondary lymphoid organs, which is predominantly mediated by NK cells. Thus, SIVagm replication is strongly controlled in $\mathrm{LN}$ with no or little viral replication in BCF, while the virus continues to replicate efficiently in the intestine ${ }^{18}$.

The reasons why SIVagm replication in the intestine does not lead to disruption of the intestinal barrier in AGM are unclear. The underlying mechanisms could be multiple. The maintenance of the gut barrier could be related to the preservation of Th17 cells in SIVagminfected $\mathrm{AGM}^{19}$. Immunoglobulins $\mathrm{A}(\lg \mathrm{A})$ are also known to play an important role in the control of intestinal inflammation ${ }^{20}$. IgA are the dominant antibody isotype found in mucosal secretions ${ }^{21}$. Secretory $\lg A(S \lg A)$ limit the penetration of commensal bacteria through the epithelium $^{22}$ achieving efficient protection of the epithelial barrier by immune exclusion ${ }^{23}$. SIgA exert an anti-inflammatory function in the gut and play a key role in the prevention of 
tissue damage and recovery from infection ${ }^{24,25}$. IgA-deficient humans indeed exhibit gut microbiota dysbiosis and inflammatory bowel disease ${ }^{26}$.

B-cell dysregulation, including in mucosa, was described early in the HIV/AIDS epidemics. Damage to the B-cell compartment in HIV infection includes loss of normal BCF architecture in LN, polyclonal hypergammaglobulinemia, increased turnover of $B$ cells, and eventually irreversible loss of memory B-cell responses with advancing HIV disease $27,28,29 . \lg \mathrm{A}$ producing $B$ cells and plasma cells are not spared from the HIV-induced damage ${ }^{27}$. There are unusually low anti-HIV IgA responses when compared to IgG responses in mucosal fluids $^{30}$. The level of inflammation markers correlates with the loss of $\lg A$ in plasma and intestine during HIV infection ${ }^{28}$. PLWH showed dysfunction of $B$ cell isotype switching, leading to limited anti-microbial IgA production ${ }^{28}$. In contrast, AGMs developed highermagnitude plasma gp120-specific IgA and IgG responses than macaques, whereas macaques developed more robust gp140-directed IgG responses ${ }^{31,32}$.

Gut-IgA antibodies are principally produced from plasma cells generated in BCF of Peyer's patches and mesenteric (mes) LN. Mesenteric LN draining the intestine amplify IgA responses, but also display high levels of viral replication in SIVmac-infected macaques (MAC, the animal model of HIV) from early stage of infection on and constitute major viral reservoirs during chronic HIV-1 and SIVmac infection ${ }^{33}$. We raise the hypothesis that the control of SIVagm replication in BCF in AGM could have a beneficial impact on the maintenance of physiological IgA levels and thereby, on regulation of inflammation in the intestine. To address this question, we analyzed NK cells, viral replication, SIgA, and isotype class switch from memory B cells in mesLN and intestine of AGM during SIVagm infection compared to SIVmac-infected macaques. Our study reveals a relation between NK cell mediated control of SIV infection and IgA levels in the mesLN, and delivers novel insights into the mechanisms that protect the mucosal tissue integrity during lentiviral infections.

\section{RESULTS}

\section{Induction of CXCR5+ NK cells in B cell follicles from mesenteric lymph nodes during SIVagm infection}

Previous studies have shown that the control of SIVagm replication in secondary lymphoid tissues $^{12,34}$ was mediated by NK cells ${ }^{17}$. So far, however, NK cells have only been studied in BCF of peripheral LN and spleen during SIVagm infection. No data are available on NK cells in mesLN in natural hosts of SIV. We thus investigated whether NK cells from mesLN upregulate CXCR5 in response to SIVagm infection in AGM, and whether they migrate into $B C F$ of mesLN. The viral replication profiles in mesLN were analyzed in situ by FISH (Fig.1A, C). The mesLN from uninfected $A G M s(n=4)$ and cynomolgus macaques (MAC, $n=4$ ) were 
compared to mesLN collected during SIVagm ( $n=4$ AGM), and SIVmac infection ( $n=4$ MAC). As expected, we did not observe SIV RNA in mesLN from non-infected animals. Signs of viral production were generally observed only in in mesLN of SIV-infected MAC but not during chronic SIVagm infection (Fig.1A, B). These data are in line with previous results showing a viral control in mesLN of SIVagm-infected $\mathrm{AGM}^{34}$.

We next investigated the localization of NK cells inside the mesLN by immunohistochemistry. We did not observe NK cells in BCF of mesLN from non-infected animals nor from infected MAC, however, NK cells were present in BCF of mesLN from SIV-infected AGM (Fig.S1A-B). NK cells were accumulating in BCF already in acute SIVagm infection (day 9 p.i. Fig. S1A-B). CXCR5 is a chemokine receptor allowing migration of $B$ cells and $T_{F H}$ into $B C F$. In line with the spatial dynamics of the NK cells, CXCR5+ NK cells were increased after SIVagm infection in mesLN of AGM as compared to non-infected animals (Fig.1D, Fig.S1C). In a previous study, we had depleted NK cells during chronic SIVagm infection through administration of anti-IL-15 ${ }^{17}$. This lead to increases in viremia and strong viral replication in peripheral LN and spleen ${ }^{17}$. Here, we analyzed in the same, anti-IL-15 treated animals, the expression of SIV RNA in situ in the mesLN and observed strong viral replication in their $\mathrm{BCF}$, demonstrating that NK cells control SIVagm replication not only in peripheral LN and spleen, as reported previously ${ }^{17}$, but also in the LN draining the intestine (Fig.1C). Altogether, these results demonstrate that CXCR5+ NK cells are rapidly induced in mesLN upon SIVagm infection and protect BCF from viral replication.

\section{Memory B cell IgA+ production is increased in mesLN during SIVagm infection}

We raised the question if there was a link between the NK cell-mediated control in mesLN and the humoral responses. We investigated $B$ cells and $\lg A$ responses in mesLN during SIVagm and SIVmac infection. Uninfected ( $n=6$ animals per species) and chronically SIVinfected animals (6 AGM, 6 MAC) were analyzed. B cells were defined as CD45+CD3CD20+ cells and memory B cells as CD45+CD3-CD20+lgD- cells (Fig.S1D). The frequency of total B cells did not vary significantly following SIVagm or SIVmac infection in mesLN or any intestinal compartment analyzed when compared to uninfected animals (Fig.S1E-F). Then, we analyzed the isotype of membrane-bound lg expressed by memory B cells from mesLN. IgA class switching is known to be altered in intestinal lymphoid tissues of chronic SIVmac and HIV-1 infections ${ }^{30,35}$. We observed no differences between non-infected and SIV-infected MAC regarding IgA+, IgG+ or IgM+ memory B cells (Fig.1E). There was also no change concerning IgG-expressing memory B cells in SIVagm-infected AGM, but an increased frequency of IgA- and IgM-expressing memory $B$ cells compared to non-infected AGMs (Fig.1E). Moreover, the frequency of $\lg A+$ and $\operatorname{lgM}+$ memory $B$ cells from mesLN in SIVagm infection correlated positively with the frequency of CXCR5+ NK cells also present in 
mesLN (Fig.1F). Of note, the frequency of IgG+ memory B cells from mesLN in SIVagm infection correlated negatively with the frequency of CXCR5+ NK cells present in mesLN (Fig.1F). These results showed that $\lg A_{+}$and $\lg M_{+}$memory $B$ cells were increased in mesLN following SIVagm infection in contrast to SIVmac infection, and suggest a link between these responses and the NK cell-mediated viral control.

\section{Loss of total IgA in the intestinal lumen during chronic SIVmac in contrast to SIVagm infection}

We then explored the total IgA response from intestine in SIV infection. We analyzed here in parallel the ileum, jejunum and colon in non-infected, acutely and chronically infected animals. The analysis of $\lg A$ distribution by immunohistochemistry demonstrated the presence of $\lg A$ (positive cells and antibodies) within the lamina propria and in the lumen of the gut from non-infected AGM and MAC (Fig.S2A). During acute infection, no differences regarding the level nor the location of $\lg A$ were observed in AGM or MAC when compared to uninfected animals (Fig.2A-C, Fig.2SA). However, the IgA staining was drastically decreased in chronic SIVmac infection compared to non-infected MAC in all intestinal compartments, unlike to chronic SIVagm-infected animals (Fig.2A-C, Fig.S2A). Scarce IgA+ cells were still detected in the lamina propria of intestinal tissues from chronic SIVmac-infected animals but massively lost from the lumen (Fig.2D). In contrast, IgA signals were detected in chronic SIVagm infection in all intestinal compartments and within the gut lumen (Fig.2D, Fig.S2A).

We further analyzed IgA expressing cells in the intestine (jejunum, ileum and colon) by quantifying Ig expressions on memory $B$ cells using flow cytometry (Fig.2E-F). In MAC, the frequency of $\mathrm{IgM}+$ memory $B$ cells remained comparable between non-infected and chronically infected animals (Fig.2E), while IgG+ memory B cells increased in jejunum and colon from MAC SIV-infected compared to uninfected animals (Fig.2E). In contrast, IgA+ memory $B$ cells decreased in colon from SIV-infected MAC compared to uninfected animals (Fig.2E). In AGM, the frequencies of $\lg G+, \lg A+$ and $\lg M+$ memory $B$ cells remained comparable between non-infected and chronically infected animals (Fig.2F). Altogether, AGM displayed normal levels of IgA production in all three intestinal compartments analyzed, and showed no increases of $\lg G$, in contrast to MAC. In MAC, IgA levels were maintained in acute infection but decreased in chronic SIVmac infection, and SIgA seemed to be particularly lost in the gut lumen.

Total secreted IgA but not IgA+ memory B cells in blood reflected the dynamics in intestine-associated tissues during SIVagm and SIVmac infections

We then investigated whether the alterations in Ig profiles observed in the gut-associated lymphoid tissues were reflected in the blood. $\lg A+$ and $\lg G+$ memory $B$ cell frequencies 
remained comparable between uninfected and infected animals in the blood, for both MAC and AGM (Fig.3A-F). Only a significant decrease of IgM+ memory B cells in AGM after chronic SIV infection was observed (Fig.3C, F). We also measured the concentrations of circulating $\lg G$ and $\lg A$ in plasma from infected $A G M$ and MAC as well as in control animals. There was no difference between non-infected and SIV-infected AGM for plasma IgA and IgG titers (Fig.3G, H). In contrast, there was a decrease of circulating IgA ( $p$-value 0.0065 ) in plasma from SIVmac-infected MAC compared to non-infected MAC (Fig.3G, H). Altogether,

\section{Inflammation in SIVmac infection correlated with lower blood IgA levels}

Because $\lg A$ are associated with microbiota control and an anti-inflammatory function ${ }^{21,24}$, we analyzed here if there was an association between $\lg A$ levels and inflammation. The concentrations of systemic markers of inflammation and microbial translocation (SCD14, LPS-binding protein (LBP) and intestinal fatty-acid binding protein (I-FABP) were quantified in the plasma by ELISA (Fig.S3A-C). These markers are used to evaluate inflammation levels through monocyte/macrophage activation ( $S C D 14)$, intestinal epithelial barrier disruption (I-FABP) and microbial translocation (sCD14, I-FABP, LBP) ${ }^{27,28}$. As expected, we detected an increased concentration of plasma SCD14 in chronic SIVmac infection as compared to baseline levels but not in SIVagm infection (Fig.S3A). Strikingly, plasma IgA and SCD14 correlated negatively in SIVmac infection $(r=-0.57 ; p=0.04)$ (Fig.3I). The two other markers, I-FABP and LBP, did not show increases in chronic SIVmac infection (Fig.S3A-C). Of note, plasma lgG, which can be a marker for hypergammaglobulinemia in HIV/SIVmac infections ${ }^{36}$ and thus for abnormal B cell response, correlated positively with LBP $(r=0.89 ; p<0.0001)$ in SIVmac infection (Fig.3J). In contrast, there was no correlation between any of these inflammatory markers and Ig levels in SIVagm infection (Fig.S4A-H). These data support the association between systemic inflammatory levels and abnormal isotypic Ig profiles in SIV infection.

\section{Stronger SIV-ENV specific memory B cell responses in mesLN and intestine during SIVagm than SIVmac infection}

We then measured the SIV-specific Ig levels. Recombinant gp140-foldon Env GP140 proteins from SIVagm and SIVmac were constructed and used for measuring gp140-specific memory B cells by flow cytometry. As expected, the frequencies of gp140-specific memory B cells were increased in blood following SIVagm and SIVmac infections as compared to uninfected animals (Fig.4A-B). The frequencies of gp140-specific memory B cells were also increased in the mesLN and in all studied intestinal compartments (ileum, jejunum, colon) in SIVagm-infected as compared to uninfected AGM. However, in contrast to blood, there were 
no detectable increases in chronic SIVmac infection in mesLN and intestinal mucosa (Fig.4C-E).

We evaluated the distribution of $\lg A+$, $\lg M+$ and $\lg G_{+}$gp140-specific memory $B$ cells in chronically-SIV infected animals in blood, mesLN and intestine. IgG+ gp140-specific memory $B$ cells were predominant in blood, while lgM+ gp140-specific memory B cells were predominant in mesLN and intestinal mucosa (Fig.S5A-D). The frequencies of $\lg A+g p 140-$ specific memory B cells ranged between $4.21 \%$ and $4.25 \%$ of median in blood and all studied gut-associated-tissues of MAC and AGM (Fig.S5A-B). Among all compartments, the frequencies of $\lg A+$ gp140-specific memory $B$ cells were highest in the jejunum of chronically SIVagm-infected AGM with $8.47 \%$ of median (Fig.S5D).

We quantified secreted gp140-specific Ig in plasma. Both MAC and AGM mounted gp140specific antibody responses following SIVmac and SIVagm infection, respectively, while the titers in non-infected animals did not increase (Fig.S5E, F). As expected, the affinities of the IgGs were higher than those of $\lg A s$.

Finally, we evaluated if there was association between the plasma gp140-specific Ig profiles and the levels of inflammation or microbial translocation (Fig.4F, G and Fig.S6A-J). There was trend for a negative correlation between anti-gp140 $\lg A$ antibodies and plasma I-FABP in SIVmac-infected macaques (Fig.4F).

\section{DISCUSSION}

Our study demonstrates major differences regarding the $\lg \mathrm{A}^{+}$memory $\mathrm{B}$ cells in the mesLN and intestine between non-pathogenic SIVagm and pathogenic SIVmac infection. Previous studies have already analyzed $\lg A$ and $B$ cell responses during SIVagm and SIVmac infections in distinct body compartments, including blood, milk, saliva, vaginal and rectal secretions ${ }^{28,36,37}$. These previous reports have shown that SIV-infected AGMs display robust Env-binding antibody responses in milk, higher than those in macaque and humans, which may contribute to the absence of postnatal transmission reported for natural SIV hosts ${ }^{38}$. Here, we investigated whether NK cells have the capacity to migrate into BCF of mesLN and control SIVagm infection. Moreover, we performed a comprehensive analysis of total and ENV-specific IgA and B cell responses in several tissues: blood, gut-associated secondary lymphoid organs (mesLN) and three intestinal compartments (ileum, jejunum, colon). Furthermore, we analyzed if there was a link between NK cells and humoral responses in the mesLN and/or between the $\lg A$ responses and systemic inflammation.

Our study uncovered that NK cells migrate into BCF of mesLN during SIVagm infection where they control viral replication, and that they accumulate already in acute infection in mesLN BCF in response to SIVagm infection. We show that $\operatorname{IgA}+$ memory $B$ cells were increased in mesLN during SIVagm infection in contrast to SIVmac infection. Moreover, 
normal intestinal IgA levels (jejunum, ileum and colon) were maintained during acute and chronic SIVagm infection, while they strongly decreased in chronically SIVmac-infected macaques compared to healthy macaques. Our data in the pathogenic SIV model are in agreement with previous reports on HIV-1 infection describing that the infection does not induce vigorous specific IgA responses in any body fluid examined ${ }^{27,30}$. Our study underlines that in contrast to pathogenic HIV-1/SIVmac infections in humans and macaques, $\lg \mathrm{A}^{+}$ memory $B$ cells in the mesLN are protected during SIVagm infection in AGM. The levels of CXCR5+ NK cells and IgA+ memory B cells in mesLN were strongly correlated, suggesting that indeed the NK-cell mediated viral control is an important factor in the protection and preserved function of the mucosal B cell responses in non-pathogenic SIV infection. The lack of chronic viral replication in BCF during SIVagm infection as opposed to untreated HIV$1 /$ SIVmac infections would avoid chronic inflammation and destruction of the anatomical architecture, in particular fibrosis and disruption of the FDC network as described during early HIV infection ${ }^{39}$, thereby protecting $\mathrm{B}$ cells in $\mathrm{BCF}^{36}$. The natural hosts thus display an original resilience mechanism. They develop a tissue-specific viral control limited to secondary lymphoid organs. This would lead to preservation of normal anti-viral adaptive immune responses, including the $\lg A$ responses in the gut. These $\lg A$ responses could then play a key role in the control of bacterial translocation and inflammation, which then participates in the maintenance of an intact epithelial barrier despite continuous replication of SIVagm. The continuous SIVagm replication in the gut allows the virus to achieve sufficiently high levels in blood to be more transmissible. Altogether, our data suggest a tissue-specific viral control with distal, indirect impact on other tissues. Our results do not exclude other nonmutually exclusive or synergistic mechanisms of protection of the intestinal tissue, such as strong repair mechanisms in $\mathrm{AGM}^{40}$, protection of Th17 cells and less IFN-g responses ${ }^{17,19,41}$. Moreover, it is not excluded that NK cells also directly modulate B cell responses, including in HIV infection ${ }^{42}$. It has been shown that NK cells can influence humoral responses by diminishing $T_{F H}$ cells and that they can also limit germinal center reaction ${ }^{43,44}$. Also, a crosstalk between NK and B cell has been described that could impact isotype class switch in $B$ cells ${ }^{45,46}$. Aberrant class switching from $\lg M$ to $\lg G$ rather than to $\lg A$ is a hallmark of chronic inflammatory diseases in the gut ${ }^{47}$. The induction of CXCR5+ NK cells capable of migrating into BCF of mesLN might facilitate the impact of NK cells on B cell responses and also reduce inflammation through the viral control.

Our data also provide a further explanation of why early ART has a benefit with regard to treatment initiated in chronic HIV infection. Since early ART is known to protect BCF structures in the intestine ${ }^{39}$, thereby most likely improving IgA responses, early ART might increase through $\lg A$ the control of chronic inflammation and also Env-specific antibody responses in the gut-associated tissues ${ }^{29}$. 
Blood did not reflect the $\lg \mathrm{A}^{+}$memory $\mathrm{B}$ cell occurring in the intestine. Indeed, the $\lg \mathrm{A}+$ and gp140-specific memory $B$ cell responses were increased in mesLN and intestine in AGM in contrast to MAC in response to SIV infection, but no difference was detected in blood between AGM and MAC. More than $90 \%$ of gut lgs are produced in situ by plasma cells in contrast to genital mucosa, where half of the lgs are coming from the blood ${ }^{48}$. This could explain why $B$ cells in blood did not accurately reflected the dynamics in the intestine and further demonstrate the importance to analyze intestinal tissue for understanding the in situ physio-pathological virus-host interactions.

The decrease of IgA signal in lumen area from chronically SIVmac-infected macaques was observed in all three intestinal compartments studied (ileum, jejunum, colon). The results we observed in the macaque resemble those previously described in HIV infection ${ }^{27,28}$. The decrease of the $\lg A$ production was predominantly due to a strong loss in the lumen, and not in the lamina propria, even if $\lg \mathrm{A}^{+}$memory $B$ cells seemed to also decrease in SIV chronically infected macaques compared to healthy macaques, at least in the colon. A major difference between non-pathogenic and pathogenic SIV infection was thus the selective maintenance of $\lg A$ in lumen during SIVagm infection. The loss of $\lg A$ in lumen might be due to decreased levels of plasma cells, the major producers of Ig. Of note, plasma cells could not be analyzed here, and also not by others in previous studies because of a lack of anti-CD19 antibodies specific for plasma cells from non-human primates. It will though be interesting in the future to investigate plasma cells and to understand their role in SIVagm infection.

It has been reported that depletion of B cells in SIVagm infection leads to moderately increased viral production in the intestine ${ }^{49,50}$. Thus, a partial, although weak contribution of $B$ cells to viral replication in the gut during SIVagm infection is not excluded and could indirectly participate to inflammatory control. However, even if AGM develop better gp120 responses, they do not seem to develop more or better circulating neutralizing antibodies than PLWH ${ }^{51}$. Total IgA showed stronger negative correlations with SCD14 and LBP in SIVmac infection, than ENV-specific IgA. Also, the ratios of total IgA/lgG were decreased in SIVmac infection, but not that of gp140-specific $\operatorname{lgA} / \mathrm{lgG}$. This suggests that the inflammatory control in SIV infection in the intestine is more associated with the maintenance of total IgA responses than with SIV-specific IgA responses. The total, non-SIV-specific IgA responses in the intestine during SIVagm infection might strongly contribute to protect against translocation of bacteria together with the other proposed mechanisms protecting the epithelial barrier.

A limitation of our study is that we did not analyze Peyer's patches. The latter are known for being major sites for induction of $\lg A+$ memory $B$ cells $^{52,53,54}$. The tissue sections we analyzed from the AGMs did not contain Peyer's patches and such an analysis was not feasible. However, B cells are known to rapidly migrate from Peyer's patches to the regional mesLN ${ }^{54}$, which we studied here. A recent study moreover revisited the role of mesLN and 
showed that mesLN and not Peyer's Patches were the major inductive site on anti-rotavirus $\lg$ A responses ${ }^{55}$.

Overall, we show that $\lg \mathrm{A}^{+}$memory $B$ cells were normal or increased in the mes $L N$ and intestine during SIVagm infection. AGMs may have developed resilience mechanisms where tissues are synergistically protected. Thus, NK cells control viral replication in LN, including mesLN as shown here. This could protect the immune response, including the SIgA production in the intestine, contributing to the maintenance of bacterial control and epithelial barrier integrity, despite viral replication in the intestinal mucosa. In contrast, mesLN are major viral reservoirs in pathogenic HIV/SIV infections ${ }^{56}$ and $\operatorname{lgA}$ responses in intestine are damaged $^{27}$.

In conclusion, our study suggests an original distal NK-cell mediated mechanism to protect $\lg \mathrm{A}$ responses. We uncovered indeed that NK cells of mesLN upregulate CXCR5 in SIVagm infection and rapidly migrate into mesLN BCF. This could have potentially important consequences on the protection of intestinal IgA responses and inflammation control. Enhancing HIV-specific IgA response in mucosa have been largely discussed in the context of vaccine studies ${ }^{27}$. Here our data suggest that total non-specific IgA contributes to the inflammatory control. Strategies allowing early and persisting viral control in BCF and/or restoring normal total $\lg A$ levels in the intestine could reveal beneficial for reducing inflammation in virologically controlled PLWH with persistent residual inflammation and in the research for therapies toward HIV cure.

\section{REFERENCES}

1. Soares, M. P., Teixeira, L. \& Moita, L. F. Disease tolerance and immunity in host protection against infection. Nat. Rev. Immunol. 17, 83-96 (2017).

2. Carlos, A. R., Weis, S. \& Soares, M. P. Cross-Talk Between Iron and Glucose Metabolism in the Establishment of Disease Tolerance. Front. Immunol. 9, (2018).

3. Sereti, I. et al. Persistent, Albeit Reduced, Chronic Inflammation in Persons Starting Antiretroviral Therapy in Acute HIV Infection. Clin. Infect. Dis. 64, 124-131 (2017).

4. Hunt, P. W., Lee, S. A. \& Siedner, M. J. Immunologic Biomarkers, Morbidity, and Mortality in Treated HIV Infection. J. Infect. Dis. 214 Suppl 2, S44-50 (2016).

5. Hunt, P. W. Very Early ART and Persistent Inflammation in Treated HIV. Clin. Infect. Dis. 64, 132-133 (2017).

6. Garcia-Tellez, T. et al. Non-human primates in HIV research: Achievements, limits and alternatives. Infect. Genet. Evol. 46, 324-332 (2016).

7. Massanella, M., Fromentin, R. \& Chomont, N. Residual inflammation and viral reservoirs: alliance against an HIV cure. Curr. Opin. HIV AIDS 11, 234-241 (2016).

8. Finzi, D. et al. Latent infection of CD4 + T cells provides a mechanism for lifelong persistence of HIV-1, even in patients on effective combination therapy. Nat. Med. 5, 512517 (1999).

9. Wong, J. K. \& Yukl, S. A. Tissue reservoirs of HIV. Curr. Opin. HIV AIDS 11, 362-370 (2016).

10. Banga, R. et al. PD-1 + and follicular helper T cells are responsible for persistent HIV- 
1 transcription in treated aviremic individuals. Nat. Med. 22, 754-761 (2016).

11. Huot, N., Rascle, P., Garcia-Tellez, T., Jacquelin, B. \& Müller-Trutwin, M. Innate immune cell responses in non pathogenic versus pathogenic SIV infections. Curr. Opin. Virol. 19, 37-44 (2016).

12. Diop, O. et al. High Levels of Viral Replication during Primary Simian Immunodeficiency Virus SIVagm Infection Are Rapidly and Strongly Controlled in African Green Monkeys. J. Virol. 74, 7538-47 (2000).

13. Estes, J. D. et al. Damaged Intestinal Epithelial Integrity Linked to Microbial Translocation in Pathogenic Simian Immunodeficiency Virus Infections. PLOS Pathog. 6, e1001052 (2010).

14. Raehtz, K. D. et al. African green monkeys avoid SIV disease progression by preventing intestinal dysfunction and maintaining mucosal barrier integrity. PLOS Pathog. 16, e1008333 (2020).

15. Bochart, R. M. et al. Microbiome Stability with Chronic SIV Infection in AIDS-resistant Sooty Mangabeys. bioRxiv 780825 (2019) doi:10.1101/780825.

16. Pandrea, I. V. et al. Acute Loss of Intestinal CD4+ T Cells Is Not Predictive of Simian Immunodeficiency Virus Virulence. J. Immunol. 179, 3035-3046 (2007).

17. Huot, N. et al. Natural killer cells migrate into and control simian immunodeficiency virus replication in lymph node follicles in African green monkeys. Nat. Med. 23, 1277-1286 (2017).

18. Huot, N., Bosinger, S. E., Paiardini, M., Reeves, R. K. \& Müller-Trutwin, M. Lymph Node Cellular and Viral Dynamics in Natural Hosts and Impact for HIV Cure Strategies. Front. Immunol. 9, (2018).

19. Favre, D. et al. Critical Loss of the Balance between Th17 and T Regulatory Cell Populations in Pathogenic SIV Infection. PLOS Pathog. 5, e1000295 (2009).

20. Leong, K. W. \& Ding, J. L. The Unexplored Roles of Human Serum IgA. DNA Cell Biol. 33, 823-829 (2014).

21. Cerutti, A., Chen, K. \& Chorny, A. Immunoglobulin Responses at the Mucosal Interface. Annu. Rev. Immunol. 29, 273-293 (2011).

22. Boullier, S. et al. Secretory IgA-Mediated Neutralization of Shigella flexneri Prevents Intestinal Tissue Destruction by Down-Regulating Inflammatory Circuits. J. Immunol. 183, 5879-5885 (2009).

23. Phalipon, A. et al. Secretory Component: A New Role in Secretory IgA-Mediated Immune Exclusion In Vivo. Immunity 17, 107-115 (2002).

24. Corthësy, B. Secretory immunoglobulin A: well beyond immune exclusion at mucosal surfaces. Immunopharmacol. Immunotoxicol. 31, 174-179 (2009).

25. Fernandez, M. I. et al. Anti-Inflammatory Role for Intracellular Dimeric Immunoglobulin A by Neutralization of Lipopolysaccharide in Epithelial Cells. Immunity 18, 739-749 (2003).

26. Catanzaro, J. R. et al. IgA-deficient humans exhibit gut microbiota dysbiosis despite secretion of compensatory IgM. Sci. Rep. 9, 13574 (2019).

27. Kulkarni, V. \& Ruprecht, R. M. Mucosal IgA Responses: Damaged in Established HIV Infection-Yet, Effective Weapon against HIV Transmission. Front. Immunol. 8, (2017).

28. Hel, Z. et al. Dysregulation of Systemic and Mucosal Humoral Responses to Microbial and Food Antigens as a Factor Contributing to Microbial Translocation and Chronic Inflammation in HIV-1 Infection. PLOS Pathog. 13, e1006087 (2017).

29. Planchais, C. et al. Early Antiretroviral Therapy Preserves Functional Follicular Helper $\mathrm{T}$ and HIV-Specific B Cells in the Gut Mucosa of HIV-1-Infected Individuals. J. Immunol. (2018) doi:10.4049/jimmunol.1701615.

30. Mestecky, J. et al. Paucity of Antigen-Specific IgA Responses in Sera and External Secretions of HIV-Type 1-Infected Individuals. AIDS Res. Hum. Retroviruses 20, 972-988 (2004).

31. Amos, J. D. et al. Rapid Development of gp120-Focused Neutralizing B Cell Responses during Acute Simian Immunodeficiency Virus Infection of African Green Monkeys. J. Virol. 89, 9485-9498 (2015). 
32. Zhang, R. et al. Envelope-specific B-cell populations in African green monkeys chronically infected with simian immunodeficiency virus. Nat. Commun. 7, 12131 (2016).

33. Rabezanahary, $\mathrm{H}$. et al. Despite early antiretroviral therapy effector memory and follicular helper CD4 T cells are major reservoirs in visceral lymphoid tissues of SIV-infected macaques. Mucosal Immunol. 13, 149-160 (2020).

34. Gueye, A. et al. Viral load in tissues during the early and chronic phase of nonpathogenic SIVagm infection. J. Med. Primatol. 33, 83-97 (2004).

35. Schäfer, F. et al. Lack of simian immunodeficiency virus (SIV) specific IgA response in the intestine of SIV infected rhesus macaques. Gut 50, 608-614 (2002).

36. Brocca-Cofano, E. et al. Pathogenic Correlates of Simian Immunodeficiency VirusAssociated B Cell Dysfunction. J. Virol. 91, (2017).

37. Amos, J. D. et al. Lack of B Cell Dysfunction Is Associated with Functional, gp120Dominant Antibody Responses in Breast Milk of Simian Immunodeficiency Virus-Infected African Green Monkeys. J. Virol. 87, 11121-11134 (2013).

38. Chahroudi, A. et al. Mother-to-infant transmission of SIV is rare in sooty mangabeys and is associated with low viremia. J. Virol. (2011) doi:10.1128/JVI.02690-10.

39. Kök, A. et al. Early initiation of combined antiretroviral therapy preserves immune function in the gut of HIV-infected patients. Mucosal Immunol. 8, 127-140 (2015).

40. Barrenas, F. et al. Macrophage-associated wound healing contributes to African green monkey SIV pathogenesis control. Nat. Commun. 10, 5101 (2019).

41. Jacquelin, B. et al. Nonpathogenic SIV infection of African green monkeys induces a strong but rapidly controlled type I IFN response. J. Clin. Invest. 119, 3544-3555 (2009).

42. Bradley, T. et al. RAB11FIP5 Expression and Altered Natural Killer Cell Function Are Associated with Induction of HIV Broadly Neutralizing Antibody Responses. Cell 175, 387399.e17 (2018).

43. Cook, K. D., Kline, H. C. \& Whitmire, J. K. NK cells inhibit humoral immunity by reducing the abundance of CD4+ $\mathrm{T}$ follicular helper cells during a chronic virus infection. $J$. Leukoc. Biol. 98, 153-162 (2015).

44. Rydyznski, C. E. et al. Affinity Maturation Is Impaired by Natural Killer Cell Suppression of Germinal Centers. Cell Rep. 24, 3367-3373.e4 (2018).

45. Lenz, P., Engelbert Gessner, J., Sautes, C. \& Schmidt, R. E. Fey-Receptor III (CD16) is Involved in NK-8 Cell Interaction. Immunobiology 196, 387-398 (1996).

46. Blanca, I. R., Bere, E. W., Young, H. A. \& Ortaldo, J. R. Human B Cell Activation by Autologous NK Cells Is Regulated by CD40-CD40 Ligand Interaction: Role of Memory B Cells and CD5+ B Cells. J. Immunol. 167, 6132-6139 (2001).

47. Thoree, V. C. et al. Related $\lg A 1$ and $\lg$ g producing cells in blood and diseased mucosa in ulcerative colitis. Gut 51, 44-50 (2002).

48. J, M., Z, M., Pd, S., Z, H. \& Rc, A. Mucosal immunology of the genital and gastrointestinal tracts and HIV-1 infection. J. Reprod. Immunol. 83, 196-200 (2009).

49. Estes, J. D. et al. Defining total-body AIDS-virus burden with implications for curative strategies. Nat. Med. 23, 1271-1276 (2017).

50. Gaufin, T. et al. Effect of B-Cell Depletion on Viral Replication and Clinical Outcome of Simian Immunodeficiency Virus Infection in a Natural Host. J. Virol. 83, 10347-10357 (2009).

51. Norley, S. G. et al. Immunological studies of the basis for the apathogenicity of simian immunodeficiency virus from African green monkeys. Proc. Natl. Acad. Sci. 87, 9067-9071 (1990).

52. Lycke, N. Y. \& Bemark, M. The regulation of gut mucosal IgA B-cell responses: recent developments. Mucosal Immunol. 10, 1361-1374 (2017).

53. Cerutti, A. \& Rescigno, M. The biology of intestinal immunoglobulin A responses. Immunity 28, 740-750 (2008).

54. Macpherson, A. J., McCoy, K. D., Johansen, F.-E. \& Brandtzaeg, P. The immune geography of IgA induction and function. Mucosal Immunol. 1, 11-22 (2008).

55. $\mathrm{Li}, \mathrm{C}$. et al. Early-life programming of mesenteric lymph node stromal cell identity by the lymphotoxin pathway regulates adult mucosal immunity. Sci. Immunol. 4, (2019). 
56. Cumont, M. C. et al. TGF- $\beta$ in intestinal lymphoid organs contributes to the death of armed effector CD8 T cells and is associated with the absence of virus containment in rhesus macaques infected with the simian immunodeficiency virus. Cell Death Differ. 14, 1747-1758 (2007).

57. Jacquelin, B. et al. Innate Immune Responses and Rapid Control of Inflammation in African Green Monkeys Treated or Not with Interferon-Alpha during Primary SIVagm Infection. PLOS Pathog. 10, e1004241 (2014).

58. C, P. et al. Optimal maturation of the SIV-specific CD8+ T-cell response after primary infection is associated with natural control of SIV. ANRS SIC study. (2019) doi:10.1101/2019.12.20.885459.

59. Huot, N. et al. Non-Human Primate Determinants of NK Cells in Tissues at steadystate and during SIV infection. Front. Immunol. 11, (2020).

60. Yang, X., Farzan, M., Wyatt, R. \& Sodroski, J. Characterization of stable, soluble trimers containing complete ectodomains of human immunodeficiency virus type 1 envelope glycoproteins. J. Virol. 74, 5716-5725 (2000).

61. Lorin, V. \& Mouquet, H. Efficient generation of human $\lg A$ monoclonal antibodies. J. Immunol. Methods 422, 102-110 (2015).

62. Ma, D. et al. Factors Associated with Siman Immunodeficiency Virus Transmission in a Natural African Nonhuman Primate Host in the Wild. J. Virol. 88, 5687-5705 (2014).

63. He, T. et al. Critical Role for the Adenosine Pathway in Controlling Simian Immunodeficiency Virus-Related Immune Activation and Inflammation in Gut Mucosal Tissues. J. Virol. 89, 9616-9630 (2015).

64. Planchais, C. \& Mouquet, H. Easy pan-detection of human IgA immunoglobulins. J. Immunol. Methods 112833 (2020) doi:10.1016/j.jim.2020.112833.

65. Planchais, C. et al. HIV-1 Envelope Recognition by Polyreactive and Cross-Reactive Intestinal B Cells. Cell Rep. 27, 572-585.e7 (2019).

66. Gnanadurai, C. W. et al. Genetic Identity and Biological Phenotype of a Transmitted/Founder Virus Representative of Nonpathogenic Simian Immunodeficiency Virus Infection in African Green Monkeys. J. Virol. 84, 12245-12254 (2010).

\section{MATERIAL AND METHODS}

\section{Monkeys, SIV infection and ethics statement}

Twenty-seven AGMs (Caribbean Chlorocebus sabaeus) were included in this study. Seven AGM were used as uninfected controls and twenty AGM were infected with SIVagm. Twentyseven cynomolgus MACs (Macaca fascicularis [MAC]) imported from Mauritius island were included in the study, out of which twelve MAC were used as uninfected controls and fifteen MACs were infected with SIVmac. Macaques with a controller genotype $(\mathrm{H} 6)$ were not included in the study. The AGMs were infected with the SIVagm.sab92018 wildtype isolate and the MAC with the SIVmac251 isolate as previously described ${ }^{17}$. The description of the animals is shown in Supplementary Table 1 A-B.

The AGM and MAC were housed in IDMIT infrastructure facilities (CEA, Fontenay-auxRoses, France) under animal facility authorization \#D92-032-02 (Prefecture des Hauts de Seine, France) and in compliance with European Directive 2010/63/EU, the French 
regulations and the Standards for Human Care and Use of Laboratory Animals, of the Office for Laboratory Animal Welfare (OLAW, assurance number \#A5826-01, US). The study was approved by the the institutional ethical committee "Comité d'Ethique en Expérimentation Animale du Commissariat à l'Energie Atomique et aux Energies Alternatives" (CEtEA \#44). Monitoring of the monkeys was under the supervision of the veterinarians in charge of the animal facilities. Animal experimental protocols were approved by the Ethical Committee of Animal Experimentation (CETEA-DSV, IDF, France; Notification 12-098). The samples from the animals included here were from animals purchased and used for other studies. These studies were approved and accredited under statement numbers A16-016, A17-059, A12006, A17-044, A13-005 and A15-035 by the ethics committee, registered and authorized under Number 44 at the French Ministry of Education and Research with the reference numbers APAFIS\#2453-2015102713323361, APAFIS\#4442-2016030818243239 and APAFIS\# 11236-2017091214402801 and APAFIS\#319-2015031314518254.02. Some results of these other studies are already published $41,57,58,59$. Animals were handled by veterinarians in accordance with national regulations (CEA Permit Number A 92-32-02) and the European Directive (2010/63, recommendation no. 9) and in compliance with the Standards for Human Care and Use of Laboratory of the Office for Laboratory Animal Welfare (OLAW, USA) under OLAW Assurance number \#A5826-01. Animals were housed in ASL3 confinement in adjoining individual cages allowing social interactions, and maintained under controlled conditions with respect to humidity, temperature, and light (12 hours light/12 hours dark cycles). Water was available ad libitum. Animals were monitored and fed once or twice daily commercial monkey chow and fruit by trained personnel. Environmental enrichment was provided including toys, novel foodstuffs, and music under the supervision of the CEA Animal Welfare Body. Experimental procedures (animal handling, viral inoculations, and samplings) were conducted after sedation with ketamine chlorhydrate (Rhone-Merieux, Lyon, France, $10 \mathrm{mg} / \mathrm{kg}$ ). Tissues were collected at necropsy: animals were sedated with ketamine chlorhydrate $10 \mathrm{mg} / \mathrm{kg}$ ) then humanely euthanized by intravenous injection of 180 $\mathrm{mg} / \mathrm{kg}$ sodium pentobarbital. The time of necropsy is indicated in Supplemental Table A, B. The sample size varied between three and nine monkeys per group ( $n=6$ animals in most experiments). Sample analyses were performed in random distributions into groups and random order, according to the tripartite harmonized International Council for Harmonization of Technical Requirements for Pharmaceuticals for Human Use (ICH) Guideline on Methodology (previously coded Q2B). The investigators were not blinded while the animal handlers were blinded to group allocation.

\section{Tissue collections and processing}


Plasma was obtained by blood centrifugation $1800 \mathrm{x} \mathrm{g}$ for 10 minutes and stored at $-80^{\circ} \mathrm{C}$. PBMCs were isolated on density gradient (Eurobio). The cells were conserved in fetal bovine serum (FBS) (Eurobio) with 10\% of DMSO (Sigma) in liquid nitrogren. The tissue samples (mesLN, Jejunum, lleum and Colon) were cut and grinded with the help of a gentleMACS Dissociator (Miltenyi Biotec). The cells were then filtered $(70 \mu \mathrm{m}$, Clearline) and stored in FBS with $10 \%$ of DMSO in liquid nitrogen.

To conserve tissues frozen in O.C.T. (Tissue-Tek), pieces were incubated in Paraformaldehyde (PFA) 4\% (AlfaAesar) overnight. The next day, they were washed 3 times in PBS with 20 minutes of incubation at room temperature. After that, they were immersed into increasing concentrations of sucrose (Sigma), i.e. $10 \%, 20 \%$ and $30 \%$ at $4{ }^{\circ} \mathrm{C}$. After these steps, the tissues were put into O.C.T. and freezed in ethanol/dry ice solution and conserved at $-80^{\circ} \mathrm{C}$.

\section{Flow cytometry staining}

For the NK and B cell panels, we saturated non-specific sites by monkey sera. Memory B cell population gating in AGM was already realized ${ }^{32}$. For the panels (Supplementary Table 2.AB), we saturated non-specific sites by FBS. Then we added the antibody mixes to the samples ( 1 million of cells). The B cell panels also included biotinylated gp140 proteins from SIVmac and SIVagm (see below) and streptavidin-PE (ThermoFisher). Cells were permeabilized using Cytofix solution (BD). The fluorescence staining was observed and captured by a flow cytometer (BD, LSR II) and DIVA software (BD). Images were analyzed using FlowJo.

\section{Immunofluorescent staining}

All tissues in O.C.T were cut at $10 \mu \mathrm{m}$ on a cryostat (LEICA CM 3050S) and put on slides (Thermo Scientific). These slides were conserved at $-20^{\circ} \mathrm{C}$. The epitope revelation protocol was realized with PBS incubation at room temperature for 15 minutes. Then, we let the slide in methanol (Fisher Chemical) at $-20^{\circ} \mathrm{C}$ during 2 hours and incubated in formaldehyde $2 \%$ at room temperature (Sigma) for 15 minutes. We saturated non-specific sites by BSA 4\% (Sigma) during 2 hours at room temperature and washed for 1 hour with PBS. The primary antibodies (Supplementary Table $3 ; 1: 200$ ) were added overnight at $4^{\circ} \mathrm{C}$. Then we washed the slide during 1 hour in PBS and we added secondary antibodies (Supplementary Table 3; 1:200) and DAPI (Supplementary Table 3; 1:1000) for 1 hour at $4^{\circ} \mathrm{C}$. We washed the slide during 1 hour in PBS and we added mounting medium (Invitrogen). We finally dropped off the cover (Fisher Scientific, Dutscher) from the slide with mounting medium. The fluorescence staining was observed and captured by a spinning-disk (Yokagawa, CellVoyager CV1000). We analyzed these images by ImageJ (Fiji). 


\section{Relative quantification by microscopy staining}

On our acquired samples by ImageJ software (Fiji) the noise was deleted via threshold establishment. The threshold was determined by background staining analysis. Then the median of intensity fluorescence adjusted without background (MFladj) was measured on our samples in channels corresponding to DAPI and the given marker to be measured, respectively. Finally, we calculated MFladjlgA/MFladjDAPI ratio on each sample.

\section{Fluorescent In Situ Hybridization}

All tissues in O.C.T were cut at $10 \mu \mathrm{m}$ on cryostat (LEICA CM 3050S) and put on slides (Thermo Scientific). These slides were conserved at $-20^{\circ} \mathrm{C}$. The epitope revelation protocol was realized by two methods. For detection of SIVmac RNA, we followed the RNAscope protocol with SIVmac251-gag probe utilization (Advanced Cell Diagnostics Europe) and ACD HybEZ Hybridization system (Advanced Cell Diagnostics Europe, 310013). For detection of SIVagm RNA, the SIVagm probe was made based on the SIVagm.sab92018 backbone, as described in a previous study ${ }^{17}$. The primary antibodies (Supplementary Table 3; $1: 200$ ) were added overnight at $4^{\circ} \mathrm{C}$. Then we washed the slide during 1 hour in PBS and added secondary antibodies (Supplementary Table 3; 1:200) and DAPI (Supplementary Table 3; 1:1000) for 1 hour at $4^{\circ} \mathrm{C}$. We washed the slide during 1 hour in PBS and we added mounting medium (Invitrogen, 00-4958-02). We finally dropped off the cover (Fisher Scientific, Dutscher) from the slide with mounting medium. The fluorescence staining was observed and captured by a spinning-disk (Yokagawa, CellVoyager CV1000). We analyzed these images by ImageJ (Fiji).

\section{Plasma IgA and IgG purification}

Plasma IgA and IgG from AGMs and Macaques were purified by batch/gravity-flow affinity chromatography using peptide M-coupled agarose (Invivogen, SanDiego, CA) and protein G Sepharose 4 fast flow beads (GE Healthcare, Chicago, IL) for IgAs and IgGs, respectively.

\section{Trimeric SIVagm and SIVmac251 gp140 proteins}

To produce g140 foldon-type trimers of SIVmac251 (GenBank\# AJP75601.1) and SIVagm (agm.Sab92018, GenBank\# ADO34206.1) Env glycoproteins, corresponding codonoptimized DNA fragments designed based on the original construct coding for uncleaved $\mathrm{YU}$ 2 trimers $^{60}$ were synthesized (Genscript), and cloned into pcDNA ${ }^{\mathrm{TM}} 3.1 / \mathrm{Zeo}^{(+)}$expression vector (Thermo Fisher Scientific). Trimeric SIV gp140 proteins were produced by transient transfection of FreeStyle ${ }^{\mathrm{TM}} 293-\mathrm{F}$ cells using the PEI method ${ }^{61}$, and purified by highperformance chromatography using the $\mathrm{Ni}$ Sepharose ${ }^{\circledR}$ Excel Resin according to 
manufacturer's instructions (GE Healthcare). Proteins were controlled for purity by SDSPAGE and NativePAGE gel staining as previously described ${ }^{61}$, and then biotinylated using BirA biotin-protein ligase bulk reaction kit (Avidity, LLC). Biotinylated SIV gp140 trimers were dialyzed against PBS using Slide-A-Lyzer®) Cassettes (35K MWCO, Thermo Fisher Scientific), and final protein concentrations were measured using a NanoDrop 2000 instrument (Thermo Fisher Scientific).

\section{Quantification of soluble proteins by ELISA}

The soluble markers of inflammation and microbial translocation in the plasma were measured by ELISA using the following commercial kits: I-FABP (MyBiosource), sCD14 (R\&D System) and LBP (Hycult Biotech). They have already been shown to cross-react with AGM samples ${ }^{62,63}$.

For quantification of circulating plasma IgA and IgG from AGMs and macaques, high-binding 96-well ELISA plates (Costar, Corning) were coated overnight with $250 \mathrm{ng} / \mathrm{well}$ of purified trimeric streptococcal IgA-binding protein $\left(\mathrm{tSAP}^{64}\right)$ or purified goat anti-human IgG antibody (Immunology Jackson ImmunoReseach, $0.8 \mathrm{mg} / \mathrm{ml}$ final) for total $\lg A$ or $\operatorname{lgG}$ titrations, respectively. After washings with $0.05 \%$ Tween 20-PBS (Washing buffer), plates were blocked $2 \mathrm{~h}$ with $2 \%$ BSA, 1mM EDTA, 0.05\% Tween 20-PBS (Blocking buffer), washed, and incubated for $2 \mathrm{~h}$ with diluted plasma (1:100 and 1:5000 in PBS for IgA and IgG titrations, respectively) and seven consecutive 1:3 dilutions in PBS. Purified plasma IgA or IgG from AGM and Mac starting at $12 \mu \mathrm{g} / \mathrm{ml}$ and seven consecutive 1:3 dilutions in PBS were used to perform the standard ELISA range. For antibody bindings to SIVagm or SIVmac envelope antigens, high-binding 96-well ELISA plates (Costar, Corning) were coated overnight with $125 \mathrm{ng} /$ well of purified SIVagm/mac envelope proteins. After washings with $0.05 \%$ Tween 20 PBS (Washing buffer), plates were blocked $2 \mathrm{~h}$ with 2\% BSA, 1mM EDTA, 0.05\% Tween 20 PBS (Blocking buffer), washed, and incubated for $2 \mathrm{~h}$ with serially diluted purified plasma $\operatorname{lgG} / \operatorname{lgA}$ antibodies starting at $50 \mu \mathrm{g} / \mathrm{ml}$ and seven consecutive 1:3 dilutions in PBS. After washings, plates were incubated for $1 \mathrm{~h}$ with goat HRP-conjugated anti-human IgG or IgA (Immunology Jackson ImmunoReseach, $0.8 \mathrm{mg} / \mathrm{ml}$ final) in Blocking buffer, washed, and revealed with HRP chromogenic substrate (ABTS solution, Euromedex). All ELISA experiments were done in duplicates at room temperature using HydroSpeed microplate washer and Sunrise microplate absorbance reader (Tecan Männedorf).

For the quantification of the titers of SIVagm and SIVmac GP140-IgA and GP140-IgG, we constructed SIVagm and SIVmac gp140-foldon Env GP140 proteins using methods as described $^{65}$. For SIVagm, we used as backbone the SIVagm.sab92018 molecular clone ${ }^{12,66}$. GP140 proteins was added and incubated on night at room temperature in plates covered by parafilm (Costar). Then, the same protocol was used like just above for IgA and IgG titer. 


\section{Statistics}

Comparisons among independent groups were conducted using nonparametric MannWhitney U-test ( $p$-value $\leq 0.05=^{*} ; p$-value $\leq 0.01=^{* *} ; p$-value $\leq 0.001=^{* * *}$ ). Correlation analysis was performed according to Spearman $r$ test. Statistical computations were performed using Prism (GraphPad, La Jolla, CA).

\section{ACKNOWLEDGMENTS}

We are grateful for the excellent contributions from the veterinarians and staff at the IDMIT Center (Benoit Delache, Jean-Marie Helies, and Raphaël Ho Tsong Fang). NH was supported by the Fondation J. Beytout and Institut Pasteur. PR was recipient of a PhD fellowship from the University Paris Diderot, Sorbonne Paris Cité and also supported by the NIH (R01Al143457) and Institut Pasteur. CaP was recipient of a Roux-Cantarini Postdoctoral Fellowship. Cy.P. was the recipient of an ANRS post-doctoral fellowship. We would like to acknowledge grant support from Sidaction and ANRS to MMT. H.M. received core grants from the Institut Pasteur, the INSERM and the Milieu Intérieur Program (ANR-10-LABX-6901), and was supported by an ANRS grant. We gratefully acknowledge the support to IDMIT from the French government: Investments for the Future program for infrastructures (PIA) through the ANR-11-INBS-0008 grant as well as from the PIA grant ANR-10-EQPX-02-01 to the FlowCyTech facility at IDMIT. We equally acknowledge the Investments for Future grant ANR-10-INSB-04 to support the UtechS Photonic Biolmaging (Imagopole) and C2RT facilities at Institut Pasteur.

\section{AUTHOR CONTRIBUTIONS}

$\mathrm{PR}, \mathrm{NH}, \mathrm{CyP}, \mathrm{HM}$ and MMT designed the experiments. PR, CyP, NH, BJ, ML and CaP performed the experiments. VC and BJ coordinated the animal studies. $\mathrm{PR}, \mathrm{CyP}, \mathrm{NH}, \mathrm{BJ}$, ASC, HM and MMT analyzed the data. BJ, ASC and MMT obtained the funding. PR and MMT wrote the manuscript and all co-authors reviewed it.

\section{COMPETING INTERESTS}

The authors declare no competing interests. 

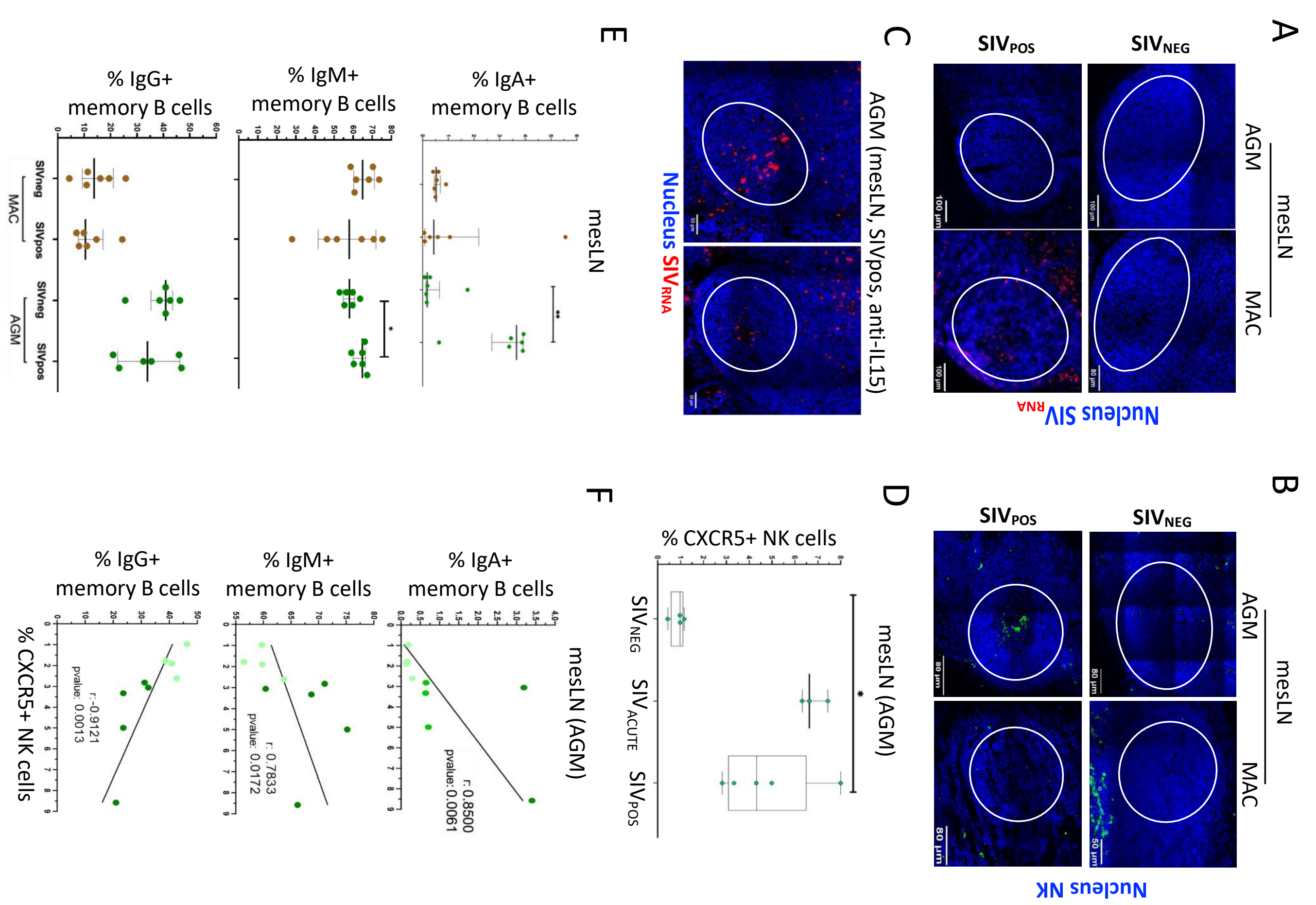
Figure 1: SIV replication, NK cells and IgA+ B cells in mesenteric lymph nodes at steady state and during SIVagm and SIVmac infection. A) Immunofluorescence staining of viral RNA in BCF from mesLN of AGM and MAC. Four uninfected and four chronically infected animals per species were studied. On the tissue sections, nucleus is stained in blue, RNASIV in red and dash white circles delineate BCF areas. B) Immunofluorescence staining of NK cells in mesLN. Four uninfected and four chronically infected animals per species were studied. Nucleus is in blue, NK cell is in green and dash white circle delineates the BCF areas. C) Immunofluorescence staining of viral RNA in mesLN from chronically SIVagm-infected AGM depleted for NK cells. The mesLN were collected from four previously described animals17. Nucleus is stained in blue, RNASIV in red. The dash white circle delineates the B cell follicle area. Two representative BCF are shown from 2 distinct animals. D) Evaluation of CXCR5+ NK cell frequencies in mesLN in non-infected AGM, at day 9 p.i. (SIV acute) and in chronic SIVagm infection (SIVpos). Percentages of CXCR5+ NK cells among total NK cells are shown. Each green circle represents one individual AGM. E) Measurement of IgA+, IgM+ and IgG+ memory B cells in mesLN from non-infected animals and from chronically infected (SIVpos) MAC (brown) and AGM (green). Percentages of among memory B cells in mesLN are shown. Each dot indicates an individual animal. F) Correlation between CXCR5+ NK cells and IgA+, IgM+ and IgG+ memory B cell in mesLN from non-infected (light green) and chronically SIVagm-infected AGM (dark green). Each green circle indicates an individual animal. Spearman $r$ test was used ( $p$-value $\leq 0.05=* ; p$-value $\leq 0.01=* * ; p$-value $\leq 0.001=* * *$ ). Animals and time points of tissue collections are described in Supplementary Table 1. 
$\% \lg \mathrm{I}+$

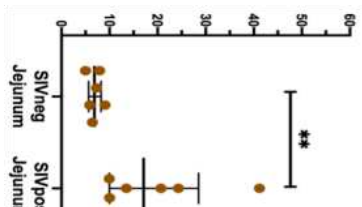

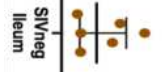

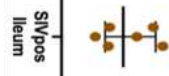

응

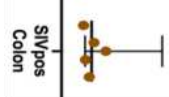

memory B cells

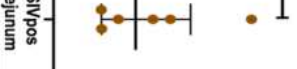

$\% \lg \mathrm{M}+$

memory B cells

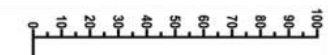

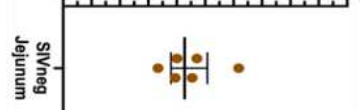

・央・

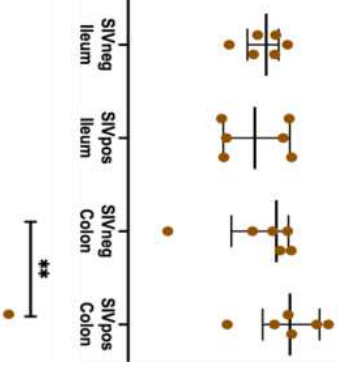

$\% \lg G+$ memory B cells 온도.

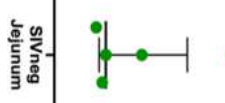

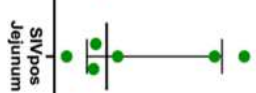

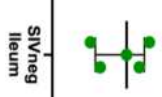

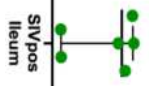

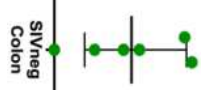

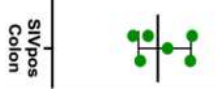

$\% \lg A+$

memory B cells

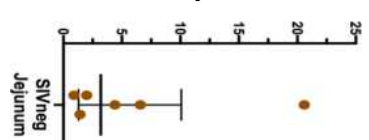

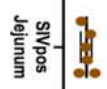

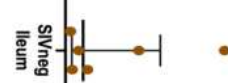

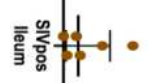

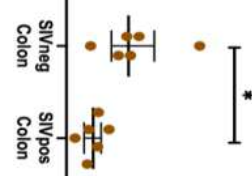

$\% \lg A+$

memory B cells memory B cells

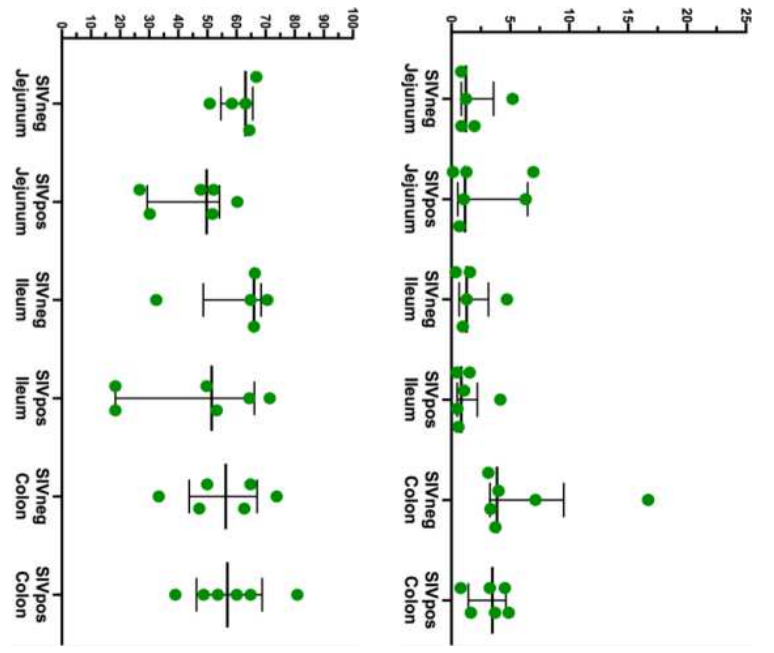

Ratio IgA signal on nucleus signal

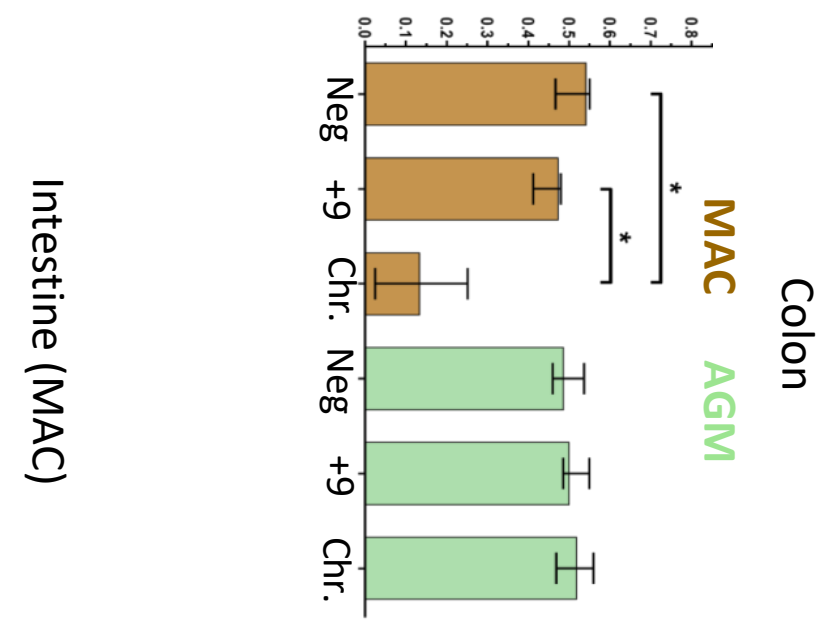

$\frac{\rho}{\circ}$

$\square$ $\cap$

Ratio IgA signal

on nucleus signal

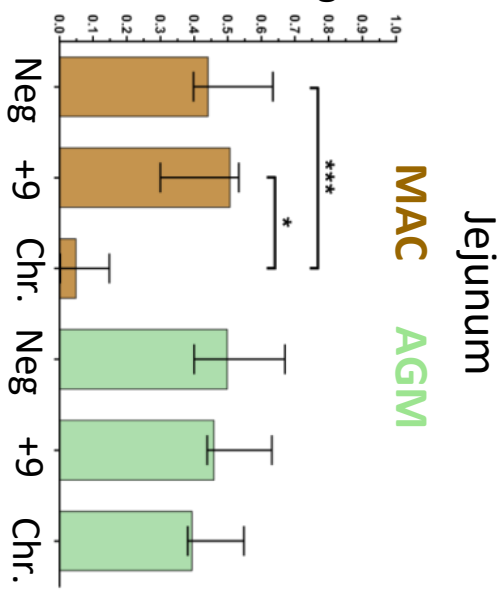

$\pi$

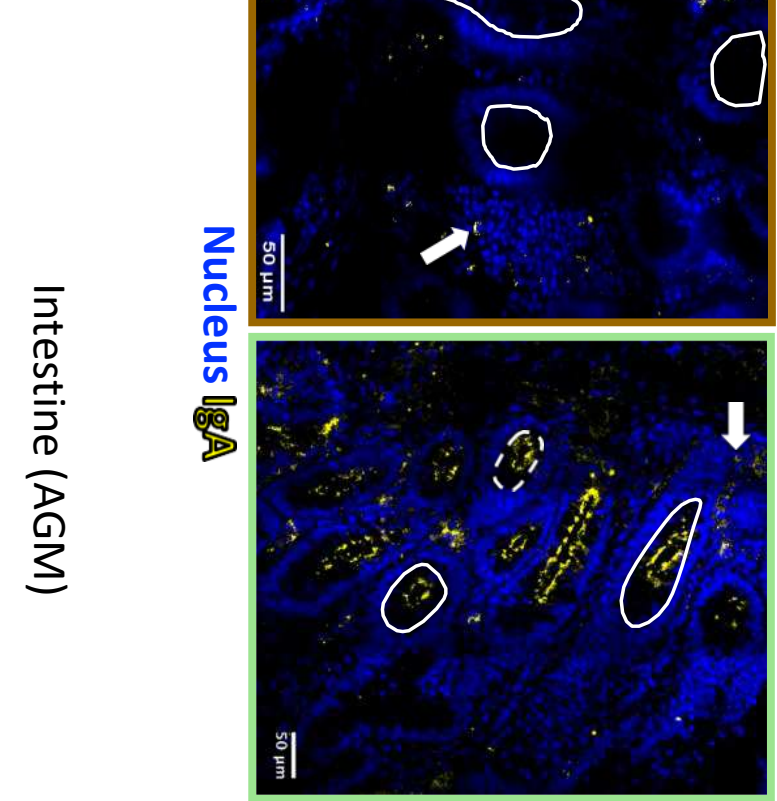

Ratio IgA signal on nucleus signal

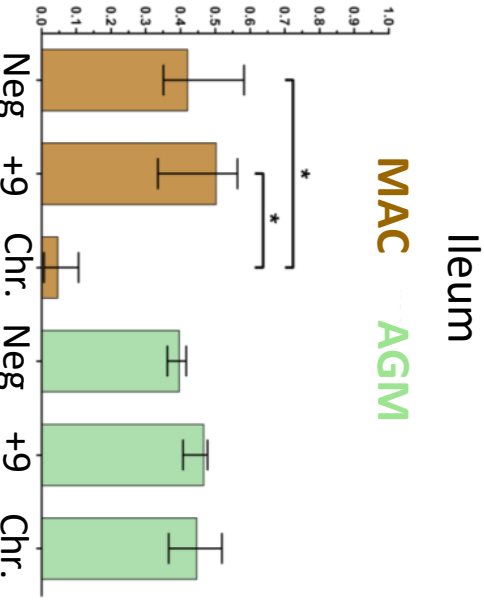


Figure 2: IgA dynamics and anatomical distributions during SIVagm and SIVmac infections in the intestine. A) Longitudinal evaluation of IgA levels in the intestine. The ratio of the IgA signal per nucleus signal was measured by microscopy in $\mathbf{A}$ ) jejunum, B) ileum and C) colon in uninfected and SIV-infected MAC (brown) and AGM (green) during acute (day 9 p.i.) and chronic SIV infection (n=3-5 animals). Welch-test was used. D) Enlargements of the immunofluorescence staining of IgA (yellow) and nucleus (blue) in the intestine from chronically SIVmacinfected MAC and chronically SIVagm-infected AGM. Arrows represent IgA+ cells and circles delineate the lumen zone ( $n=3-5$ animals per species). The images show a representative section in the ileum for one MAC and one AGM. E) Percentage of IgA+, IgM+ and IgG+ memory $B$ cells in three compartments of the intestine (jejunum, ileum and colon) from MAC (non-infected and chronically SIVmac-infected) ( $\mathrm{n}=6$ animals). F) Percentage of $\lg A+$, IgM+ and $\lg G+$ memory $B$ cells in three compartments of the intestine (jejunum, ileum and colon) from $A G M$ (non-infected and chronically SIVmac-infected) ( $n=6$ animals). Mann-Whitney U-test was used ( $p$-value $\leq 0.05=* ; p-v a l u e \leq 0.01={ }^{* *} ; p-$ value $\left.\leq 0.001=^{* * *}\right)$. 
() $\% \operatorname{lgA}+$ memory B cells

$\lg A(\mu \mathrm{g} / \mathrm{mL})$
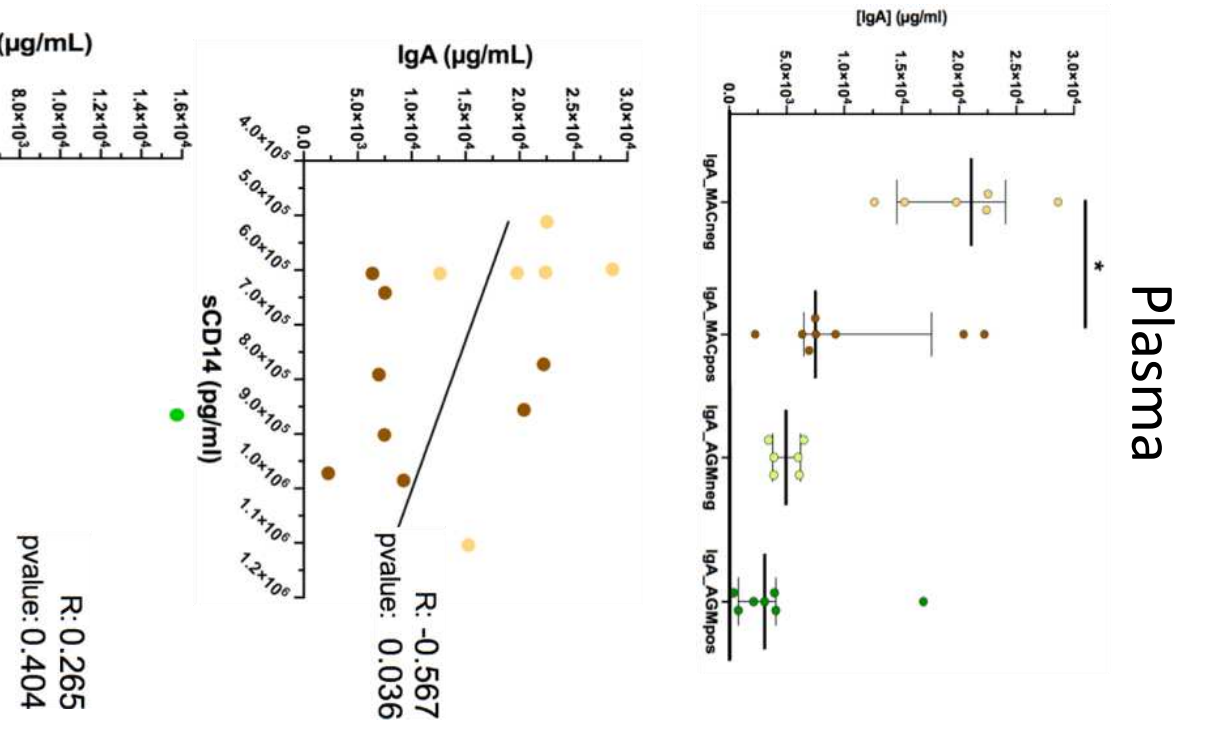

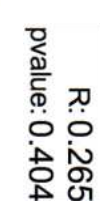
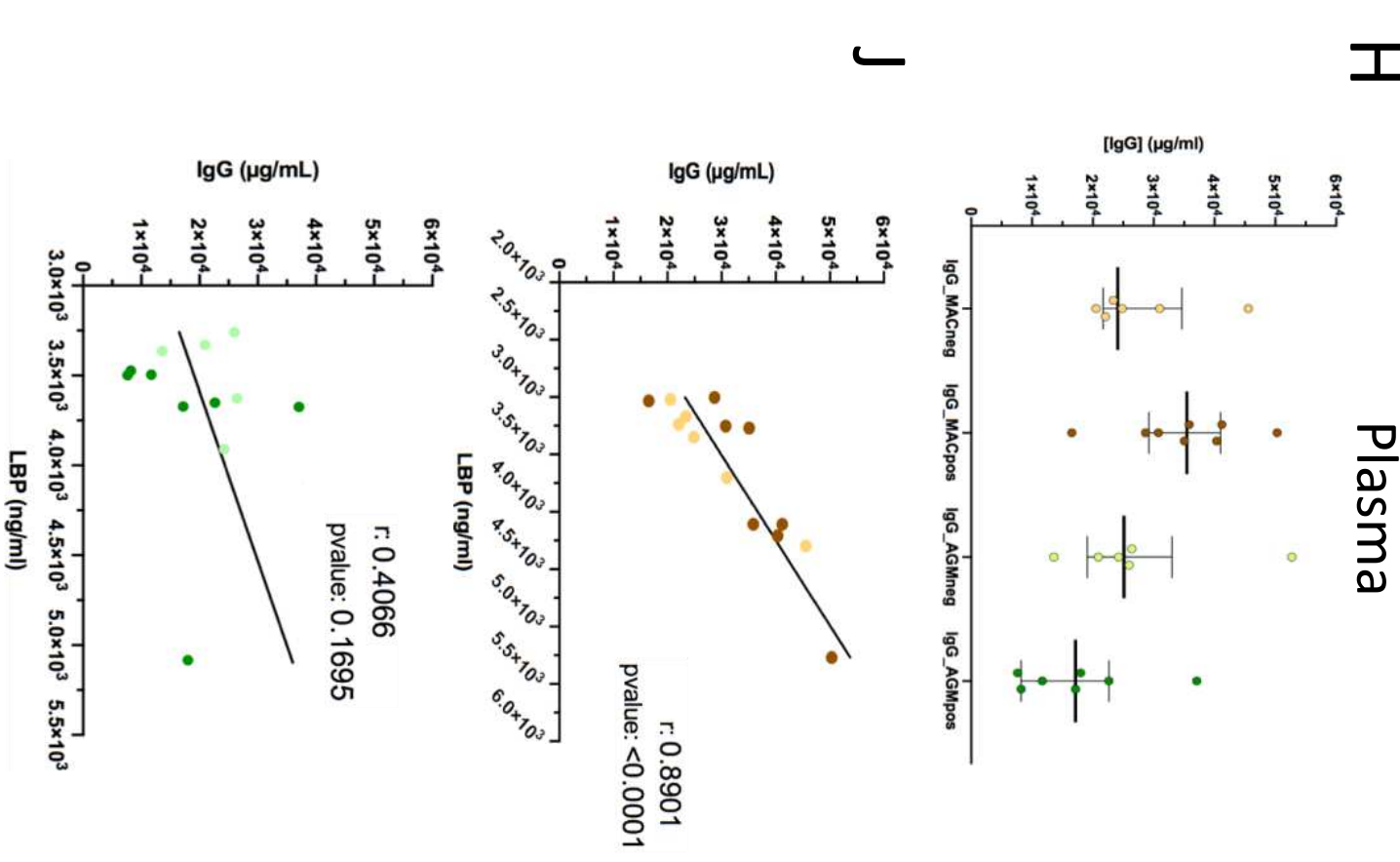

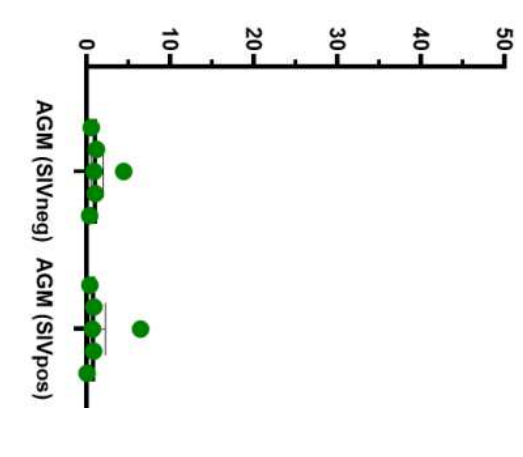

$\% \lg A+$ memory B cells

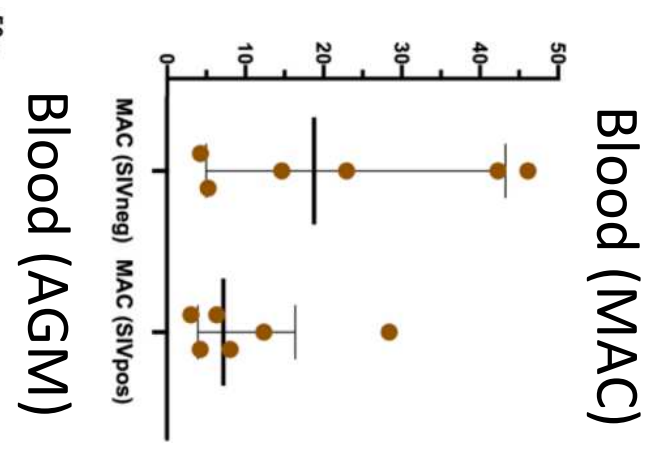

\% IgG+ memory B cells ${ }^{\Pi \pi}$ IgG+ memory B cells

$\infty$

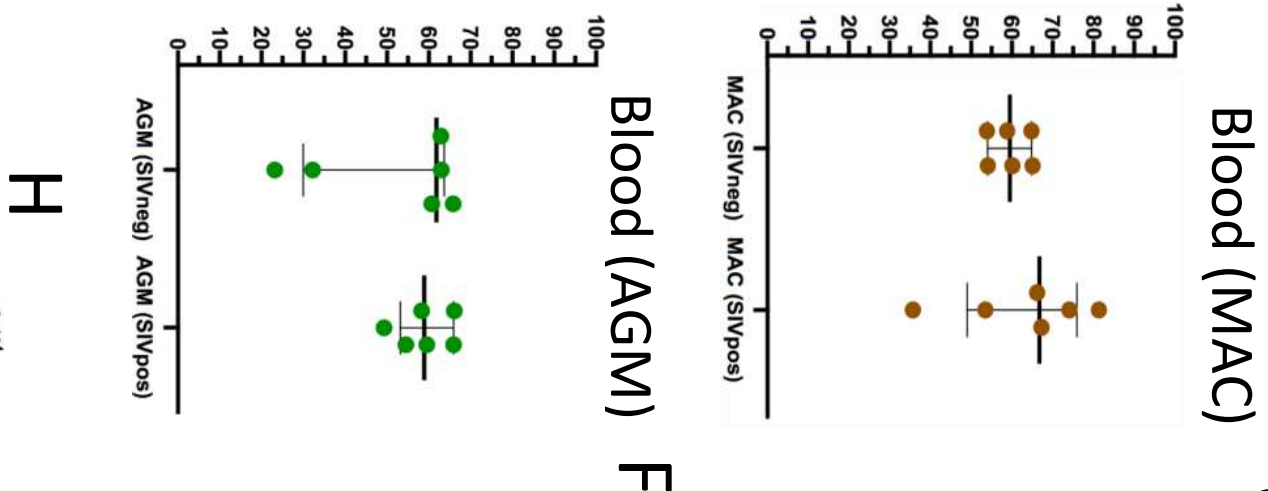

$\%$ IgM+ memory B cells $\%$ IgM+ memory B cells

$\Omega$

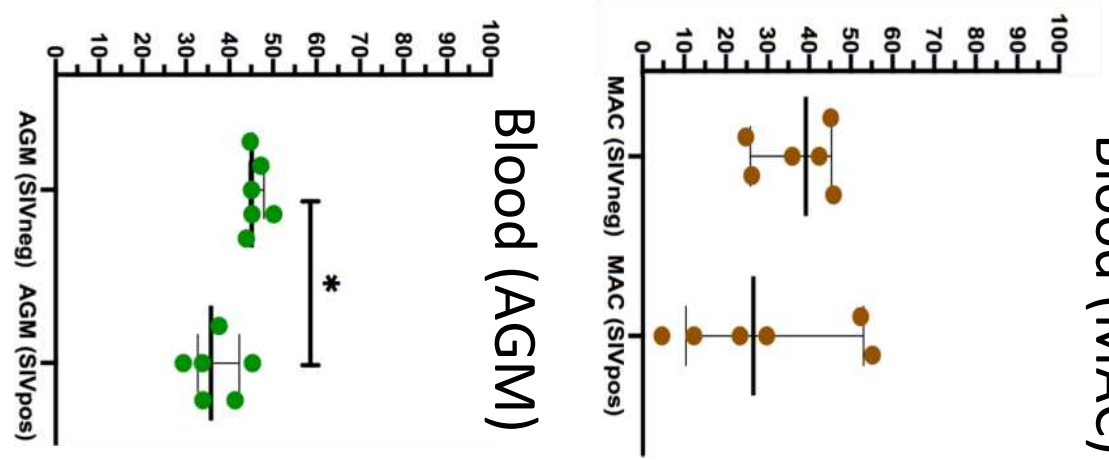


Figure 3: IgA titers in blood during SIVagm and SIVmac infections. A-C) Percentages of $\lg A+$, $\lg G+$ and $\lg M+$ memory $B$ cells in blood from MAC (non-infected and chronically SIVmac-infected) ( $n=6$ animals). D-F) Percentage of $\lg A+$, IgG+ and IgM+ memory $B$ cells in blood from AGM (non-infected and chronically SIVagm-infected) ( $n=6$ animals). G) IgA titers in plasma from MAC and AGM (non-infected and chronically SIV-infected) ( $n=6-8$ animals). H) IgG titers in plasma from MAC and AGM (non-infected and chronically SIV-infected) ( $n=6-8$ animals). Mann-Whitney U-test was used. I) Correlation between microbial translocation marker sCD14 in plasma and plasmatic-lgA in MAC (non-infected animals in light brown and chronically infected animals in dark brown) (n=6-8 animals) and in AGM (non-infected animals in light green and chronically infected animals in dark green) ( $n=6$ animals). J) Correlation between inflammatory and plasmatic-lgG in MAC (noninfected animals in light brown and chronically infected animals in dark brown) ( $n=6-8$ animals) and in AGM (non-infected animals in light green and chronically infected animals in dark green) ( $n=6$ animals). Spearman $r$ test was used ( $p$-value $\leq 0.05={ }^{*} ; p$-value $\leq 0.01={ }^{* *} ; p$ value $\left.\leq 0.001=^{* * *}\right)$. Each dot represents an individual animal. 
Figure 4: SIV ENV-specific memory B cells in blood, mesenteric lymph nodes and intestinal tissues during SIVmac and SIVagm infections. A) Percentage of gp140-specific memory B cells in blood from MAC (non-infected and chronically infected) ( $\mathrm{n}=6$ ). B) Percentage of gp140-specific memory B cells in blood from AGM (non-infected and chronically infected) ( $n=6)$. C) Percentage of gp140-specific memory B cells in mesLN from AGM (non-infected and chronically infected) and MAC (non-infected and chronically infected) ( $\mathrm{n}=6$ animals per species). D) Percentage of gp140-specific B cells in the intestine from MAC (non-infected and chronically infected) ( $n=6$ animals). E) Percentage of gp140-specific B cells in the intestine from AGM (non-infected and chronically infected) ( $n=6$ animals). Mann-Whitney U-test was used. F-G) Correlation between anti-gp140 IgA levels in plasma and I-FABP in F) MAC ( $n=6$ animals) and G) AGM ( $n=6$ animals). Spearman $r$ test was used ( $p$-value $\leq 0.05=^{*} ; p$-value $\leq 0.01=^{* *} ; p$-value $\leq 0.001=^{* * *}$ ). 
A $\quad \operatorname{AGM}\left(m L N, \mathrm{sIV}_{+9}\right)$

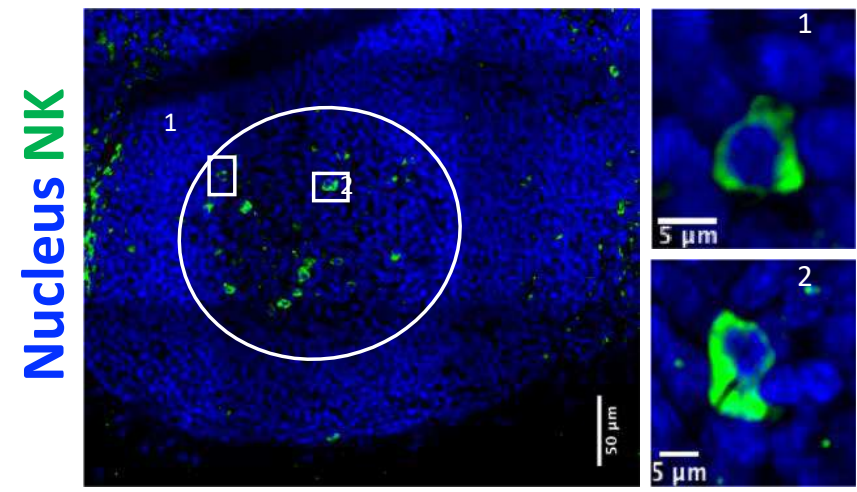

C
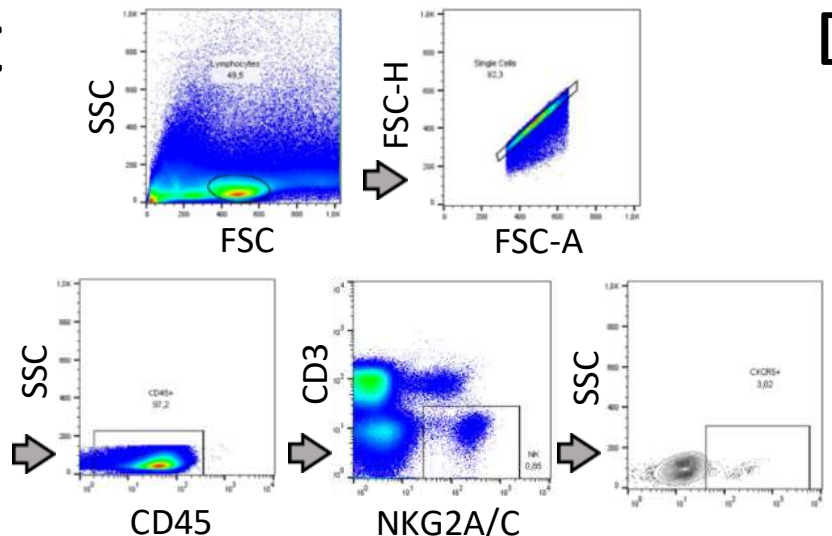

CXCR5

.

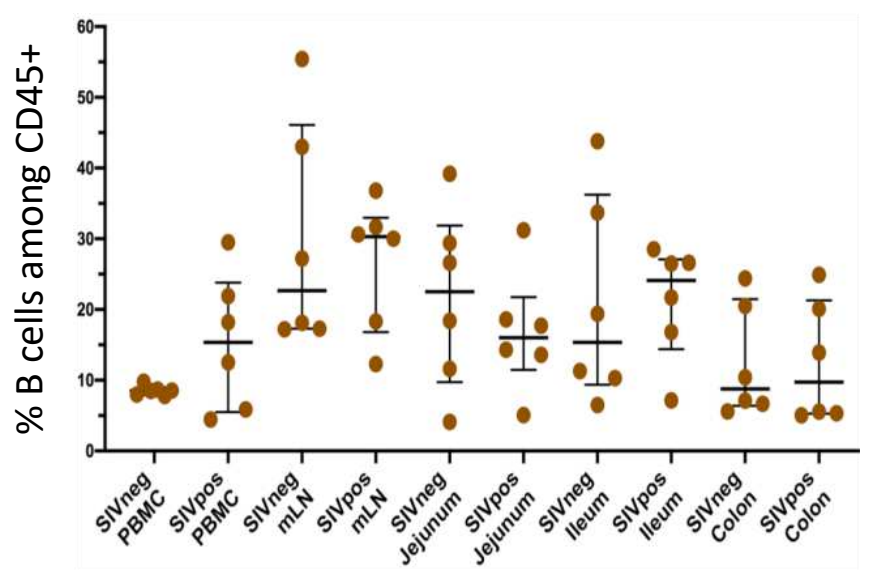

B

$\mathrm{MAC}\left(\mathrm{mLN}, \mathrm{SIV}_{+9}\right)$
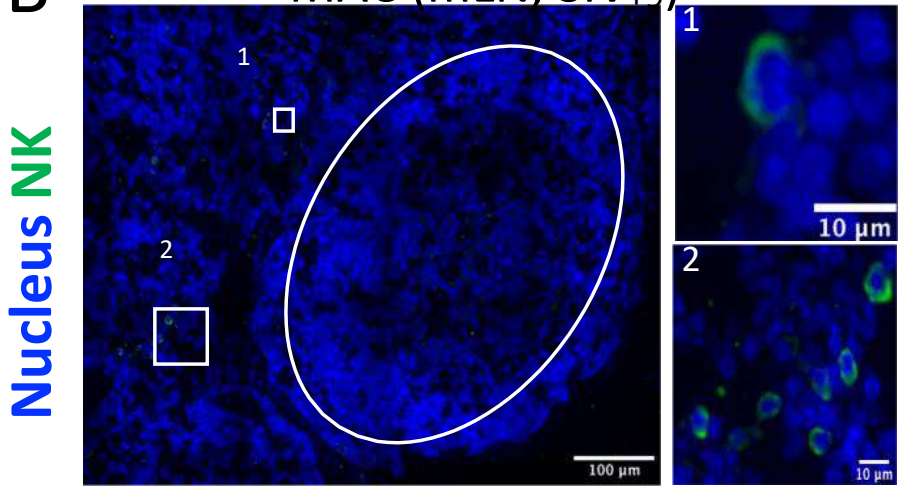

D

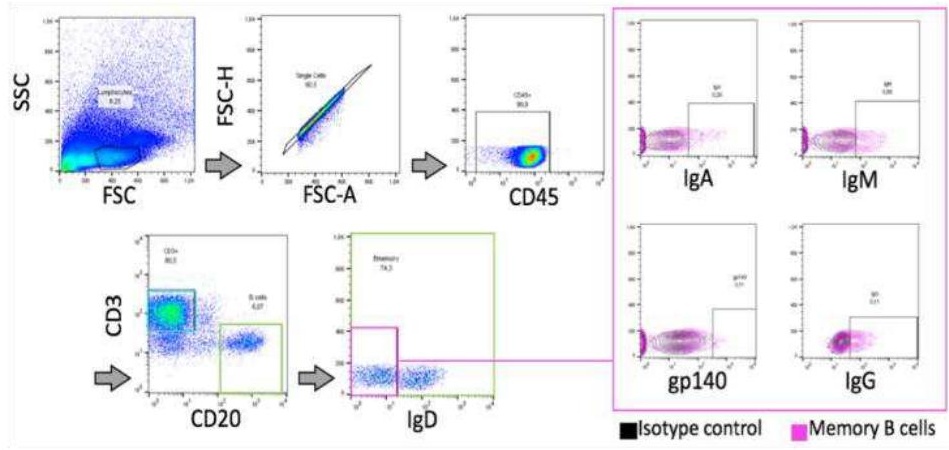

AGM

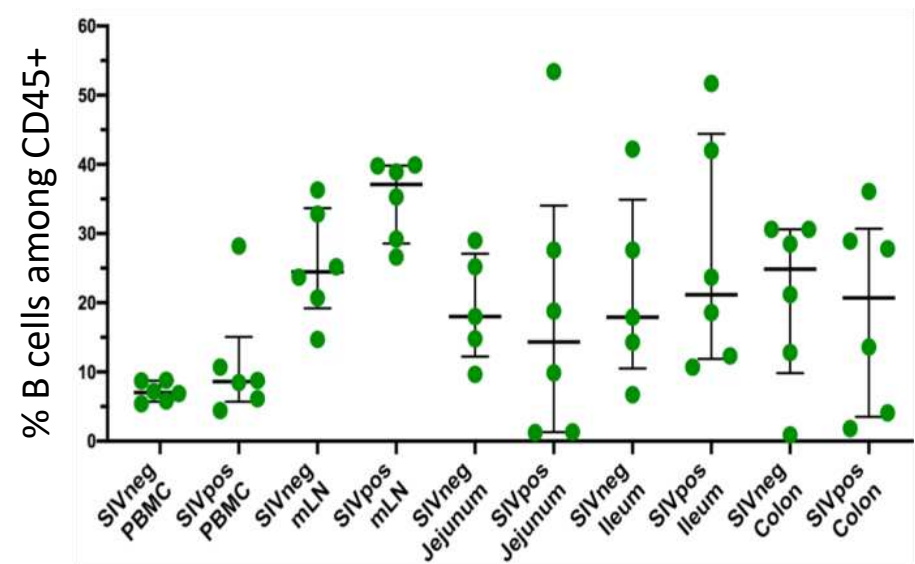


Supplementary Figure 1: A) Immunofluorescence staining of NK cells in mesLN during acute SIVagm infection. Three animals per species were analyzed at day 9 p.i. Nucleus is stained in blue, NK cells in green. The dash white circle delineates the B cell follicle area. B) Immunofluorescence staining of NK cells in mesLN during acute SIVmac infection. Three animals per species were analyzed at day 9 p.i. Nucleus is stained in blue, NK cells in green. The dash white circle delineates the B cell follicle area. C) A dot plot showing CXCR5+ NK cells. NK cells in AGM were gated as usual for NHP as CD45+CD3-NKG2A/C+ ${ }^{17}$. D) Gating strategy used for identifying IgG+/IgA+/IgM+/gp140+ memory B cells in MAC and AGM. E) Percentage of total B cells in distinct compartments (blood, mesLN, jejunum, ileum and colon) in uninfected and chronically SIVmac-infected MAC. F) Percentage of total B cells in distinct compartments (blood, mesLN, jejunum, ileum and colon) in uninfected and chronically SIVagm-infected AGM. 
Supplementary Figure 2: IgA, IgM and IgG production in the intestine during SIVagm and SIVmac infections. A) Immunofluorescence staining of IgA in the intestine (jejunum) from MAC (non-infected and chronically SIVmac-infected) and AGM (non-infected and chronically SIVagm-infected) ( $n=3-5$ animals per species). Representative sections for one animal per species are shown. 
A

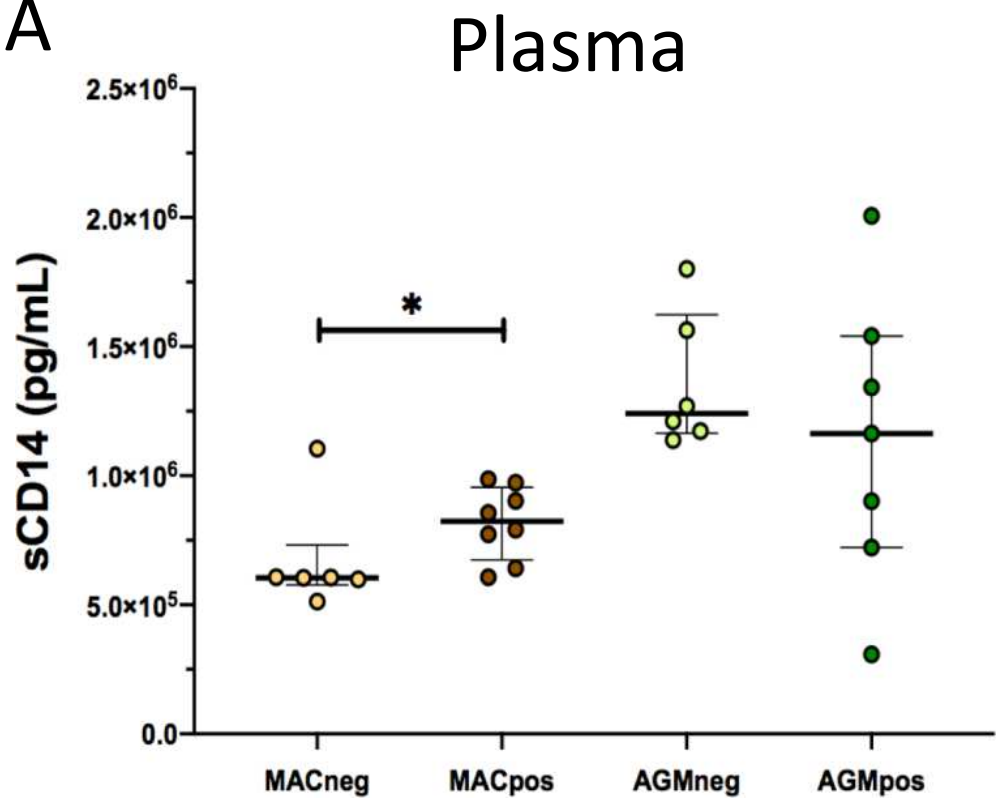

B

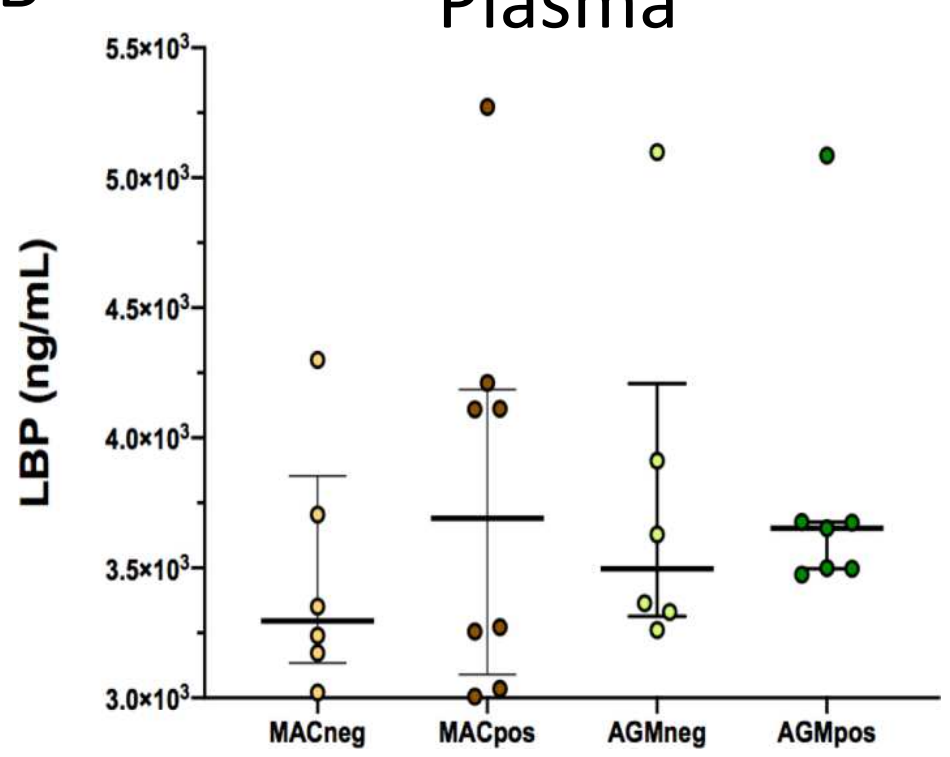

C

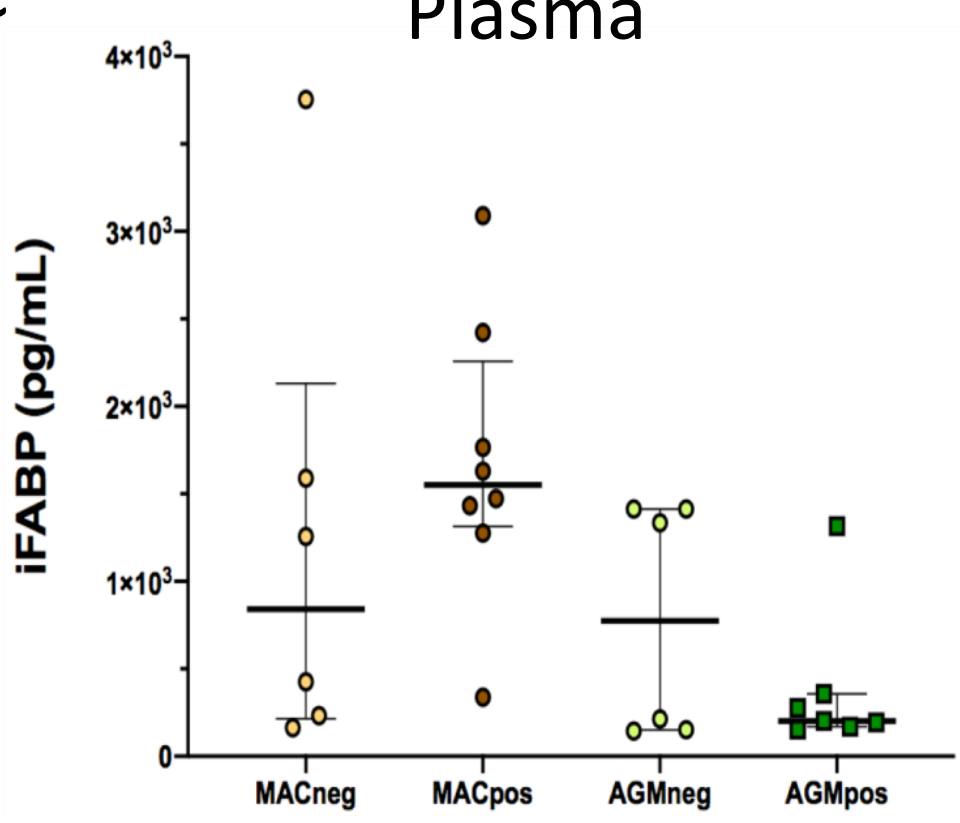


Supplementary Figure 3: Plasma titers of A) SCD14, B) LBP and C) I-FABP from uninfected and chronically SIV-infected MAC and AGM ( $n=6-8$ animals per species). Mann-Whitney U-test was used. Each dot represents an individual animal. 


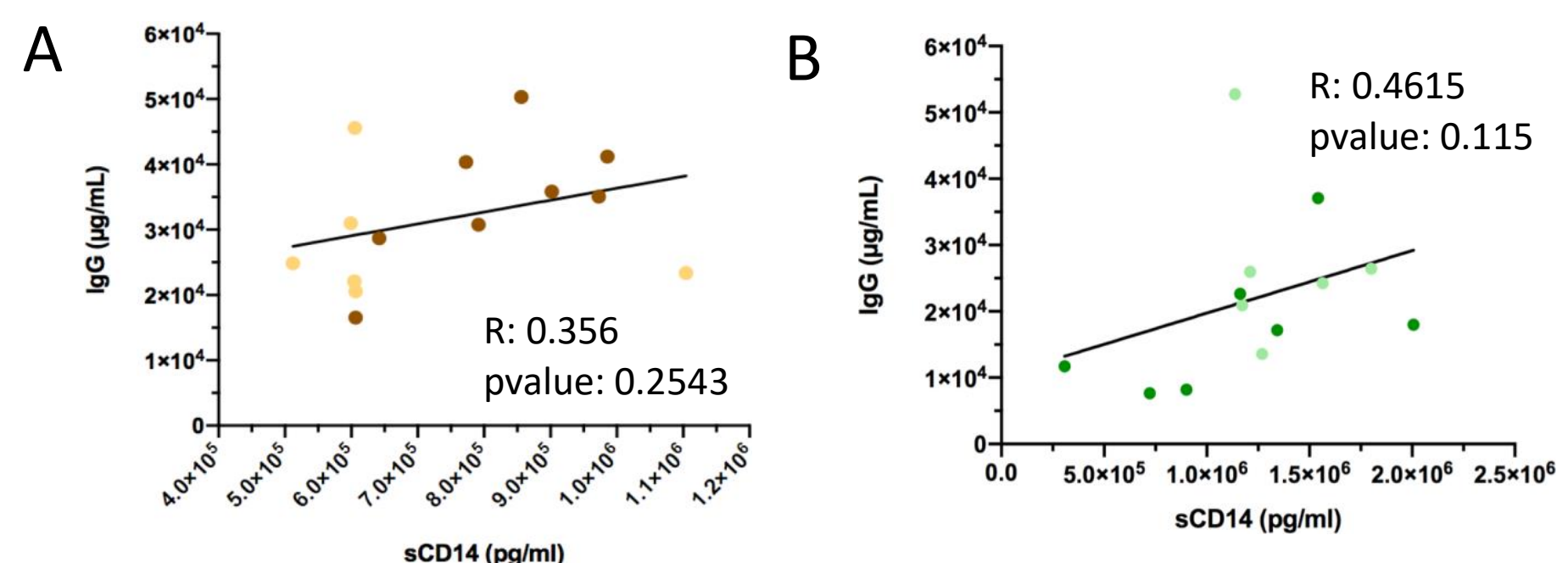

C

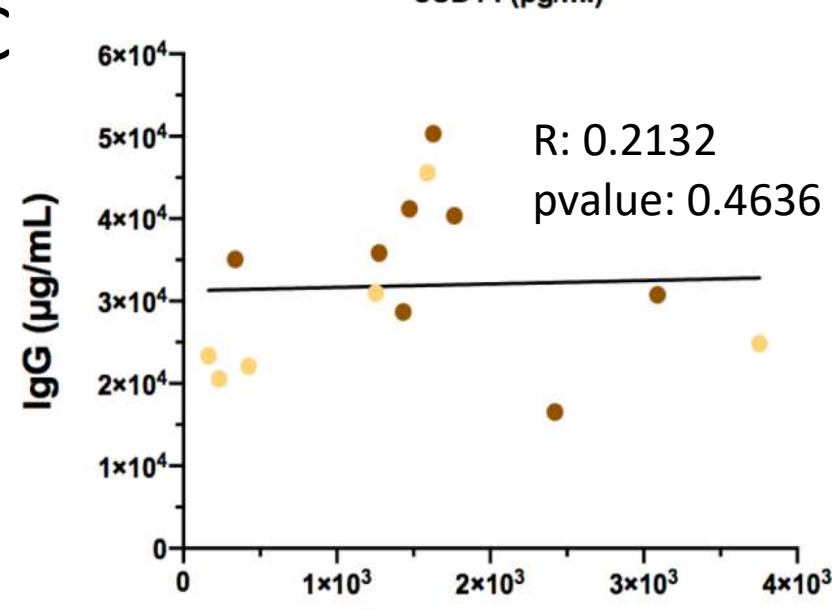

D

iFABP (pg/ml)

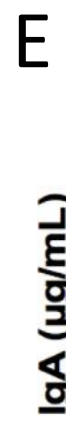

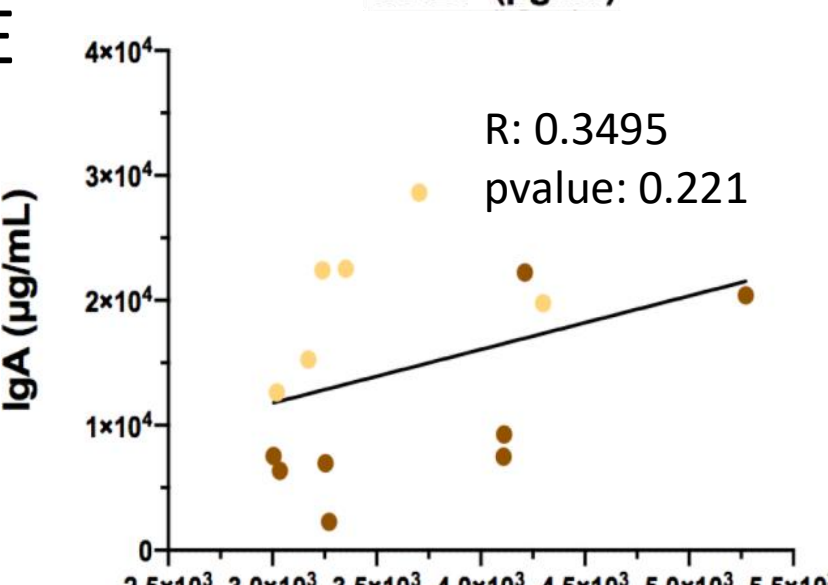

LBP (ng/ml)

동

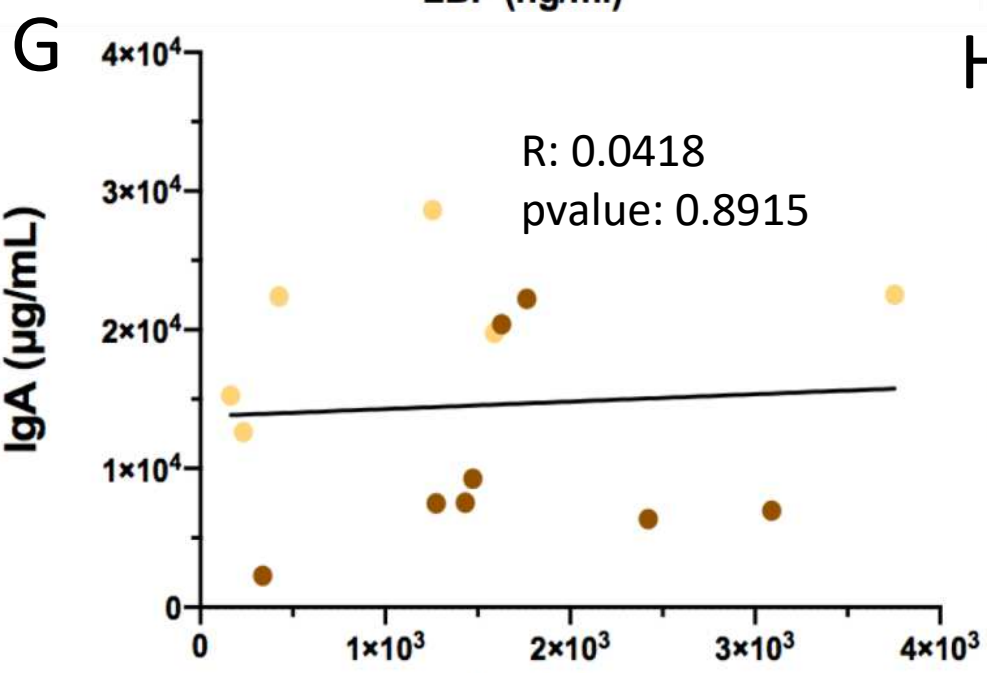

iFABP (pg/ml)

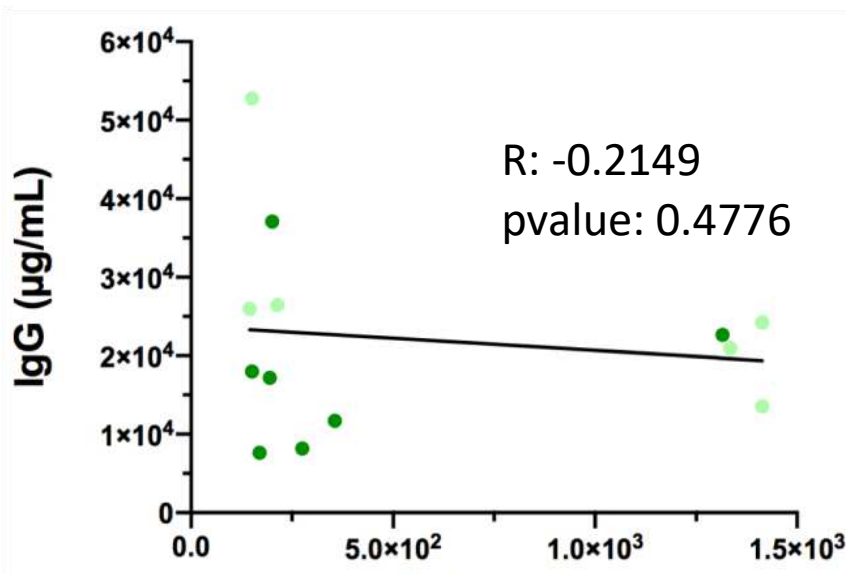

r

iFABP (pg/ml)

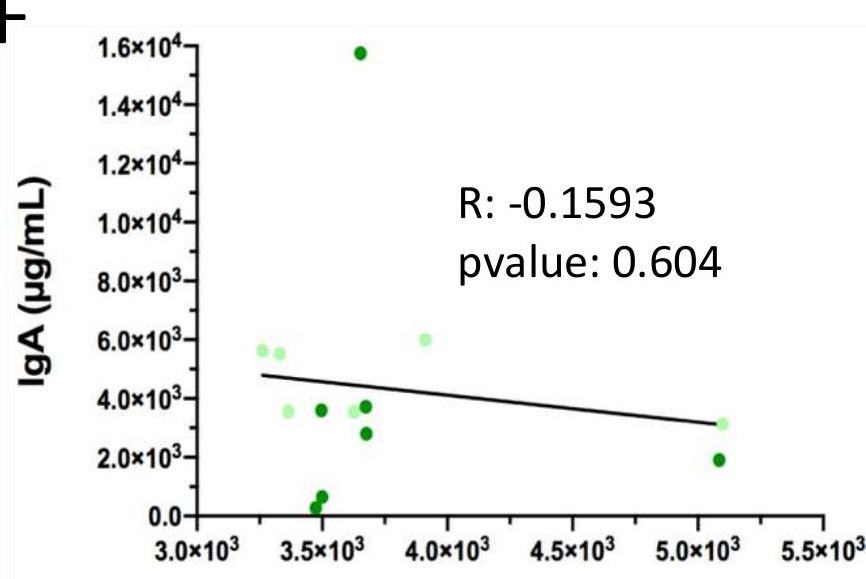

LBP (ng/ml)

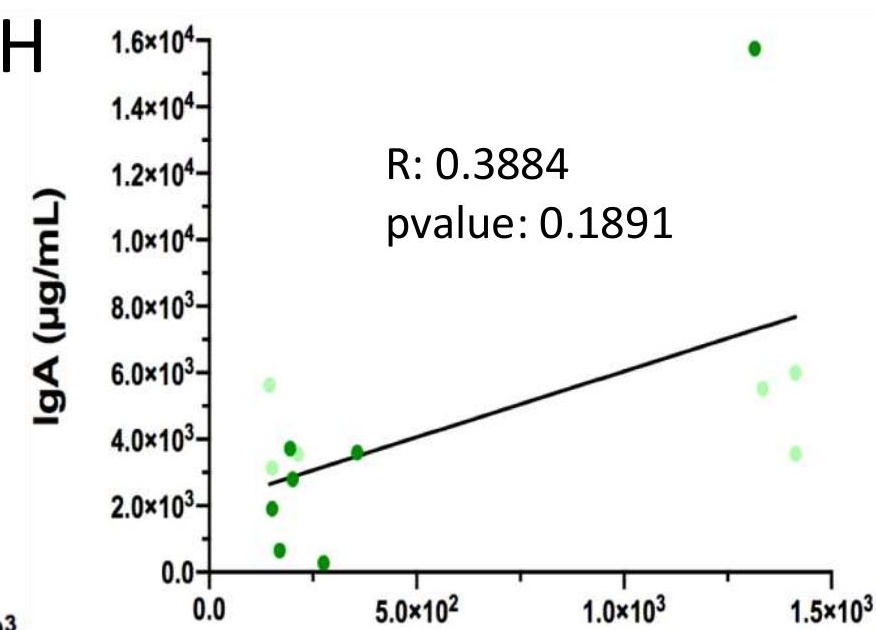

$\operatorname{iFABP}(\mathrm{pg} / \mathrm{ml})$ 
Supplementary Figure 4: Analyses of the correlations between plasmatic titers of IgG or IgA and soluble inflammation or microbial translocation markers in blood. A) Plasmatic IgG antibody levels were plotted against SCD14 in MAC. Non-infected MAC are in light brown and chronically infected in dark brown. B) The levels of IgG antibodies were plotted against those of sCD14 in AGM. Non-infected AGM are in light green and chronically infected in dark green. C) Plasmatic IgG antibody levels s were plotted against I-FABP in MAC. Non-infected MAC are in light brown and chronically infected in dark brown. D) Plasmatic IgG antibody levels were plotted against I-FABP in AGM. Non-infected AGM are in light green and chronically infected in dark green. E) Plasmatic IgA antibody levels were plotted against LBP in MAC. Non-infected MAC are in light brown and chronically infected in dark brown. F) Plasmatic IgA antibody levels were plotted against LBP in AGM. Non-infected AGM are in light green and chronically infected in dark green. G) Plasmatic IgA antibody levels were plotted against and I-FABP in MAC. Non-infected MAC are in light brown and chronically infected in dark brown. H) Plasmatic IgA antibody levels were plotted against I-FABP in AGM. Non-infected AGM are in light green and chronically infected in dark green. Each dot indicates an individual animal. Spearman $r$ test was used. 
OD $405 \mathrm{~nm}$

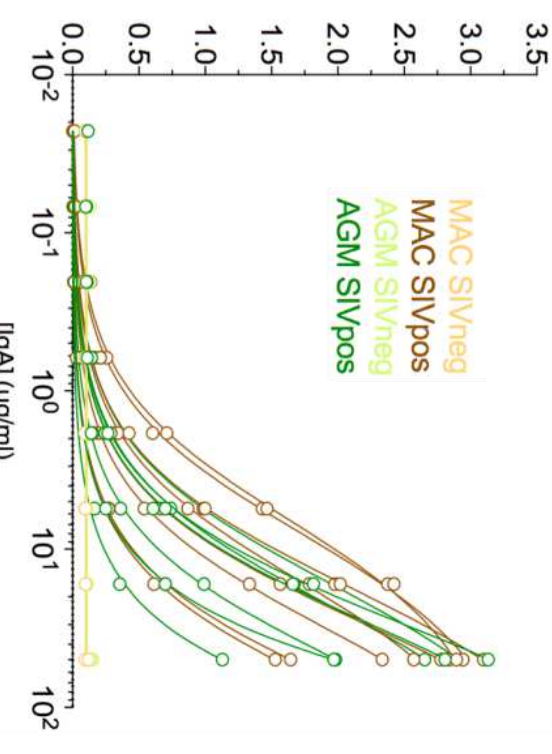

OD $405 \mathrm{~nm}$

品

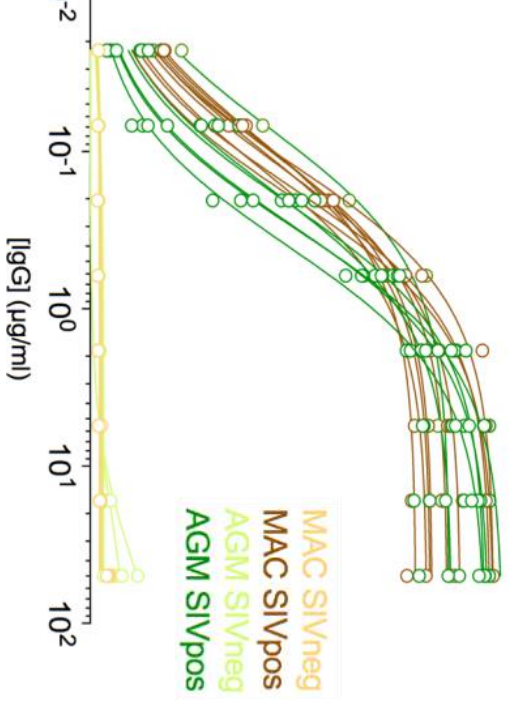

$\%$ of Ig repartition in gp $140_{\mathrm{agm}}+\mathrm{B}$ memory cells
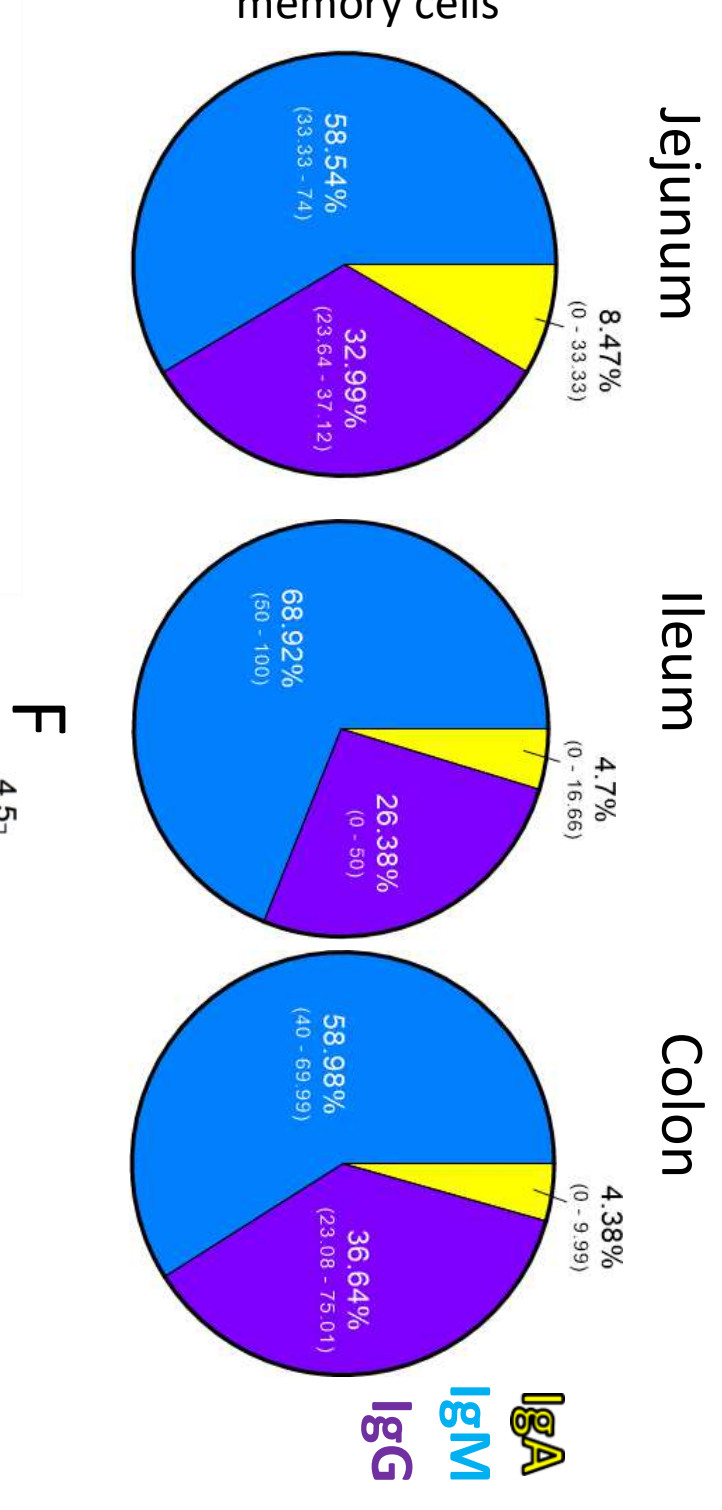

$\%$ of Ig repartition

in gp $140_{\mathrm{mac}}+\mathrm{B}$ memory cells

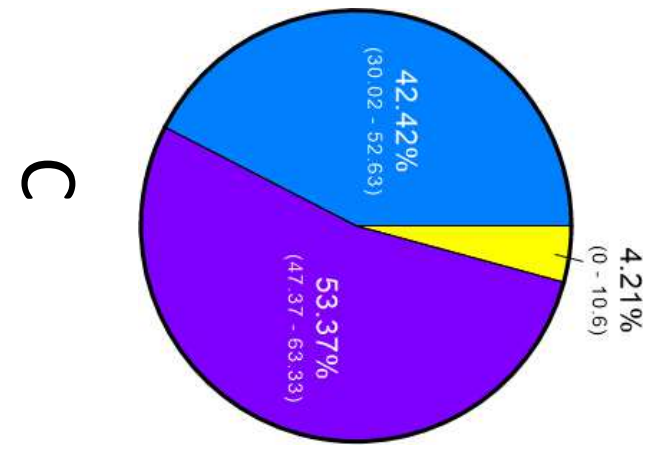

$\frac{0}{0}$
$\frac{2}{2}$
$\frac{1}{3}$
3
0
0
$\frac{1}{2}$
$\frac{D}{3}$

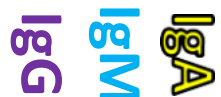

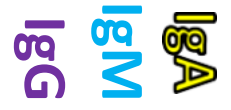

$\%$ of Ig repartition in $g p 140_{\mathrm{agm}}+\mathrm{B}$ memory cells

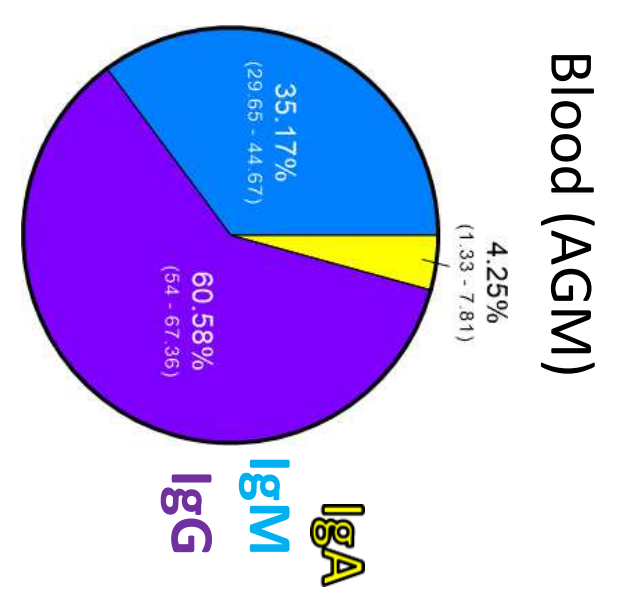


Supplementary Figure 5: A-E) Pie representation of median percentages of gp140+ IgA+, IgG+ and IgM+ memory B cells. The range (min-max) is indicated in brackets. A) GP140+ IgA+, IgG+ and IgM+ memory B cells from blood in chronically SIVmac-infected MAC ( $n=6)$. B) GP140+ IgA+, IgG+ and IgM+ memory B cells from blood in chronically SIVagm-infected AGM ( $n=6)$. C) GP140+ IgA+, IgG+ and IgM+ memory B cells from mesLN in chronically SIVagm-infected AGM (n=6). D) GP140+ IgA+, IgG+ and IgM+ memory B cells from intestine (jejunum, ileum and colon) of chronicallySIVagm-infected AGM ( $n=6)$. E) Binding of plasmatic SIV-specific IgA antibodies to trimeric gp140 proteins measured by ELISA. The green dotted line corresponds to the AGM (light green represents non-infected monkeys and dark green represents chronically infected monkeys, $n=6-7$ animals). The brown dotted line corresponds to the MAC (light brown represents non-infected monkeys and dark brown represents chronically infected monkeys, $n=6-8$ animals). F) ELISA graphs show the binding of plasmatic SIV-specific IgG antibodies to trimeric gp140 proteins. The green dotted line corresponds to the AGM (light green represents non-infected monkeys and dark green represents chronically infected monkeys, $n=6-7$ animals). The brown dotted line corresponds to the MAC (light brown represents non-infected monkeys and dark brown represents chronically infected monkeys, $n=6-8$ animals). 
Supplementary Figure 6: Search for potential correlations between SIVagm and SIVmac Env-specific IgA and IgG in blood and markers of microbial translocation and systemic inflammation. (A-F) Plasma titers of gp140-specific IgG were plotted against SCD14, LBP or I-FABP concentrations in plasma of SIVmac infected MAC (brown) and SIVagm infected AGM (green). Each dot indicates an individual animal. (G-J) Plasma titers of gp140-specific IgA were plotted against I-FABP, SCD14 or LBP concentrations in plasma of SIVmac infected MAC (brown) and SIVagm infected AGM (green). Each dot indicates an individual animal. Spearman $r$ test was used. 


\begin{tabular}{|c|c|c|c|c|c|c|c|c|c|}
\hline \multirow[b]{2}{*}{ Animals \# } & \multirow[b]{2}{*}{ Specie } & \multicolumn{5}{|c|}{ Tissues } & \multirow[b]{2}{*}{$\begin{array}{c}\text { SIV } \\
\text { (copies/mL plasma) }\end{array}$} & \multirow[b]{2}{*}{ Day Post Infection } & \multirow[b]{2}{*}{ References (DOI) } \\
\hline & & PBMCs & mesLN & Jejunum & Ileum & Colon & & & \\
\hline AGM1 & Chlorocebus sabaeus & $\mathrm{x}$ & $\mathrm{x}$ & $\mathrm{x}$ & $\mathbf{x}$ & $\mathbf{x}$ & Negative & NA & $10.1038 / \mathrm{s} 41467-021-21402-1$ \\
\hline AGM2 & Chlorocebus sabaeus & $\mathbf{x}$ & $\mathbf{x}$ & $x$ & $\mathbf{x}$ & $x$ & Negative & NA & 10.1186/s13148-020-00971-w \\
\hline AGM3 & Chlorocebus sabaeus & $\mathbf{x}$ & $\mathbf{x}$ & $\mathrm{x}$ & $\mathbf{x}$ & $\mathbf{x}$ & Negative & NA & $10.1038 / \mathrm{s} 41467-021-21402-1$ \\
\hline AGM4 & Chlorocebus sabaeus & $\mathbf{x}$ & $\mathrm{x}$ & $\mathrm{x}$ & $\mathbf{x}$ & $\mathbf{x}$ & Negative & NA & $10.1038 / s 41467-021-21402-1$ \\
\hline AGM5 & Chlorocebus sabaeus & $\mathrm{x}$ & $\mathbf{x}$ & $\mathrm{x}$ & $\mathbf{x}$ & $\mathbf{x}$ & Negative & NA & $10.1038 / \mathrm{s} 41467-021-21402-1$ \\
\hline AGM6 & Chlorocebus sabaeus & $\mathbf{x}$ & $\mathbf{x}$ & $\mathbf{x}$ & $\mathbf{x}$ & $\mathbf{x}$ & Negative & NA & $10.1038 / s 41467-021-21402-1$ \\
\hline AGM7 & Chlorocebus sabaeus & $\mathrm{x}$ & $\mathbf{x}$ & $\mathrm{x}$ & $\mathbf{x}$ & $\mathbf{x}$ & Negative & NA & $10.1186 / \mathrm{s} 13148-020-00971-\mathrm{w}$ \\
\hline AGM8 & Chlorocebus sabaeus & NA & $\mathbf{x}$ & $\mathbf{x}$ & $\mathbf{x}$ & $\mathrm{x}$ & $7.7 \times 10^{5}$ & 9 & $10.1038 / \mathrm{s} 41467-021-21402-1$ \\
\hline AGM9 & Chlorocebus sabaeus & NA & $x$ & $x$ & $\mathbf{x}$ & $\mathrm{x}$ & $3.5 \times 10^{6}$ & 9 & $10.1038 / \mathrm{s} 41467-021-21402-1$ \\
\hline AGM10 & Chlorocebus sabaeus & NA & $\mathbf{x}$ & $\mathrm{x}$ & $\mathbf{x}$ & $\mathrm{x}$ & $2 \times 10^{7}$ & 9 & $10.1038 / \mathrm{s} 41467-021-21402-1$ \\
\hline AGM11 & Chlorocebus sabaeus & NA & $x$ & NA & NA & NA & $1.4 \times 10^{4}$ & 459 & $10.1172 / \mathrm{JCl} 40093$ \\
\hline AGM12 & Chlorocebus sabaeus & NA & $\mathbf{x}$ & NA & NA & NA & $7.5 \times 10^{5}$ & 459 & $10.1172 / \mathrm{JCl} 40093$ \\
\hline AGM13 & Chlorocebus sabaeus & NA & $x$ & NA & NA & NA & $2.2 \times 10^{4}$ & 715 & $10.1186 / \mathrm{s} 13148-020-00971-\mathrm{w}$ \\
\hline AGM14 & Chlorocebus sabaeus & NA & NA & NA & $\mathbf{x}$ & NA & $2.8 \times 10^{4}$ & 64 & $10.1186 / \mathrm{s} 13148-020-00971-\mathrm{w}$ \\
\hline AGM15 & Chlorocebus sabaeus & NA & $x$ & NA & NA & NA & $6.5 \times 10^{5}$ & 402 & 10.1371/journal.ppat.1004241 \\
\hline AGM16 & Chlorocebus sabaeus & NA & $x$ & NA & NA & NA & $5.7 \times 10^{5}$ & 402 & 10.1371/journal.ppat.1004241 \\
\hline AGM17 & Chlorocebus sabaeus & NA & NA & NA & $\mathbf{x}$ & NA & $1 \times 10^{4}$ & 64 & $10.1186 / s 13148-020-00971-w$ \\
\hline AGM18 & Chlorocebus sabaeus & $\mathbf{x}$ & $\mathbf{x}$ & $\mathbf{x}$ & NA & $\mathrm{x}$ & $1.2 \times 10^{4}$ & 295 & $10.1038 / \mathrm{nm} .4421$ \\
\hline AGM19 & Chlorocebus sabaeus & $\mathbf{x}$ & $\mathbf{x}$ & $\mathbf{x}$ & $\mathbf{x}$ & $\mathbf{x}$ & $1.2 \times 10^{4}$ & 317 & $10.1038 / \mathrm{nm} .4421$ \\
\hline AGM20 & Chlorocebus sabaeus & NA & NA & $x$ & $\mathbf{x}$ & $\mathbf{x}$ & $2.3 \times 10^{4}$ & 317 & $10.1038 / \mathrm{nm} .4421$ \\
\hline AGM21 & Chlorocebus sabaeus & NA & NA & $x$ & $\mathbf{x}$ & $\mathbf{x}$ & $2.5 \times 10^{4}$ & 276 & $10.1038 / \mathrm{nm} .4421$ \\
\hline AGM22 & Chlorocebus sabaeus & $\mathrm{x}$ & NA & NA & NA & NA & $5.6 \times 10^{4}$ & 250 & $10.1038 / \mathrm{nm} .4421$ \\
\hline AGM23 & Chlorocebus sabaeus & $\mathrm{x}$ & NA & $\mathrm{x}$ & $\mathbf{x}$ & $\mathbf{x}$ & $5 \times 10^{5}$ & 792 & 10.1371/journal.ppat.1004241 \\
\hline AGM24 & Chlorocebus sabaeus & $\mathbf{x}$ & NA & $\mathbf{x}$ & $\mathbf{x}$ & NA & $1.3 \times 10^{6}$ & 779 & 10.1371/journal.ppat.1004241 \\
\hline AGM25 & Chlorocebus sabaeus & $x$ & NA & $x$ & $\mathbf{x}$ & $\mathbf{x}$ & $1.9 \times 10^{6}$ & 787 & 10.1371/journal.ppat.1004241 \\
\hline AGM26 & Chlorocebus sabaeus & NA & $\mathbf{x}$ & $\mathrm{x}$ & $\mathbf{x}$ & $\mathrm{x}$ & $4.7 \times 10^{5}$ & 65 & 10.1371/journal.ppat.1004241 \\
\hline AGM27 & Chlorocebus sabaeus & NA & $\mathbf{x}$ & NA & NA & NA & $2.2 \times 10^{5}$ & 245 & 10.1371/journal.ppat.1004241 \\
\hline
\end{tabular}

NA: not applicable

\begin{tabular}{|c|c|c|c|c|c|c|c|c|c|}
\hline \multirow[b]{2}{*}{ Animals \# } & \multirow[b]{2}{*}{ Specie } & \multicolumn{5}{|c|}{ Tissues } & \multirow[b]{2}{*}{$\begin{array}{c}\text { SIV } \\
\text { (copies/mL plasma) }\end{array}$} & \multirow[b]{2}{*}{ Day Post Infection } & \multirow[b]{2}{*}{ References (DOI) } \\
\hline & & PBMCs & mesLN & Jejunum & Ileum & Colon & & & \\
\hline MAC1 & Macaca fascicularis & $\mathrm{x}$ & $\mathrm{x}$ & $x$ & $\mathbf{x}$ & $\mathrm{x}$ & Negative & NA & 1 \\
\hline MAC2 & Macaca fascicularis & $\mathrm{x}$ & $\mathbf{x}$ & $\mathbf{x}$ & $\mathbf{x}$ & $\mathbf{x}$ & Negative & NA & 1 \\
\hline MAC3 & Macaca fascicularis & $\mathbf{x}$ & $\mathbf{x}$ & $\mathbf{x}$ & $\mathbf{x}$ & $x$ & Negative & NA & 1 \\
\hline MAC4 & Macaca fascicularis & $x$ & $x$ & $x$ & $\mathrm{x}$ & $x$ & Negative & NA & 1 \\
\hline MAC5 & Macaca fascicularis & $x$ & $x$ & $x$ & $x$ & $x$ & Negative & NA & 1 \\
\hline MAC6 & Macaca fascicularis & $\mathrm{x}$ & $x$ & $x$ & $\mathbf{x}$ & $x$ & Negative & NA & 1 \\
\hline MAC7 & Macaca fascicularis & NA & $x$ & $x$ & $\mathrm{x}$ & NA & Negative & NA & $10.1038 / \mathrm{s} 41541-020-0175-8$ \\
\hline MAC8 & Macaca fascicularis & NA & $x$ & $x$ & $x$ & $x$ & Negative & NA & $10.1038 / \mathrm{s} 41541-020-0175-8$ \\
\hline MAC9 & Macaca fascicularis & NA & $\mathrm{x}$ & NA & NA & NA & Negative & NA & 1 \\
\hline MAC10 & Macaca fascicularis & NA & NA & $\mathbf{x}$ & $\mathbf{x}$ & $\mathbf{x}$ & Negative & NA & $10.1038 / \mathrm{s} 41541-020-0175-8$ \\
\hline MAC11 & Macaca fascicularis & NA & NA & $x$ & NA & NA & Negative & NA & 1 \\
\hline MAC12 & Macaca fascicularis & NA & NA & $x$ & NA & NA & Negative & NA & $10.1038 / \mathrm{s} 41541-020-0175-8$ \\
\hline MAC13 & Macaca fascicularis & NA & $x$ & $x$ & $\mathbf{x}$ & $x$ & $9.9 \times 10^{5}$ & 9 & $10.1038 / \mathrm{s} 41467-021-21402-1$ \\
\hline MAC14 & Macaca fascicularis & NA & $x$ & $x$ & $x$ & $x$ & $1.2 \times 10^{6}$ & 9 & $10.1038 / \mathrm{s} 41467-021-21402-1$ \\
\hline MAC15 & Macaca fascicularis & NA & $x$ & $x$ & $x$ & $x$ & $1.9 \times 10^{6}$ & 9 & $10.1038 / \mathrm{s} 41467-021-21402-1$ \\
\hline MAC16 & Macaca fascicularis & $\mathbf{x}$ & $x$ & $x$ & $\mathbf{x}$ & $x$ & $3.3 \times 10^{4}$ & 262 & $10.1038 / \mathrm{nm} .4421$ \\
\hline MAC17 & Macaca fascicularis & $x$ & $\mathrm{x}$ & $x$ & NA & $x$ & $2.5 \times 10^{4}$ & 260 & $10.1038 / \mathrm{nm} .4421$ \\
\hline MAC18 & Macaca fascicularis & $x$ & $x$ & $x$ & NA & $x$ & $3 \times 10^{2}$ & 288 & $10.1159 / 000499841$ \\
\hline MAC19 & Macaca fascicularis & $x$ & $\mathbf{x}$ & $x$ & $x$ & $x$ & $1.2 \times 10^{4}$ & 365 & 1 \\
\hline MAC20 & Macaca fascicularis & $x$ & $x$ & $x$ & $x$ & $x$ & $1.9 \times 10^{4}$ & 365 & 1 \\
\hline MAC21 & Macaca fascicularis & $x$ & NA & $x$ & $\mathrm{x}$ & NA & $2.6 \times 10^{4}$ & 224 & $10.1038 / \mathrm{nm} .4421$ \\
\hline MAC22 & Macaca fascicularis & NA & $x$ & $x$ & $x$ & NA & $2.5 \times 10^{6}$ & 220 & $10.1038 / \mathrm{nm} .4421$ \\
\hline MAC23 & Macaca fascicularis & NA & NA & $x$ & NA & $x$ & Positive & 190 & 1 \\
\hline MAC24 & Macaca fascicularis & NA & NA & NA & $\mathrm{x}$ & NA & $2 \times 10^{4}$ & 530 & $10.1101 / 2019.12 .20 .885459$ \\
\hline MAC25 & Macaca fascicularis & NA & NA & $x$ & NA & $\mathbf{x}$ & $2.2 \times 10^{4}$ & 90 & 1 \\
\hline MAC26 & Macaca fascicularis & NA & NA & $x$ & $x$ & $x$ & $5.3 \times 10^{5}$ & 90 & 1 \\
\hline
\end{tabular}

Supplementary Table 1: Tissues collection and SIV infection profiles from different animals. (A) Table showing immunological parameters of 27 African Green Monkeys noninfected or infected with SIVagm.sab92018 and (B) Table showing immunological parameters of 26 cynomolgus macaques non-infected or infected with SIVmac251. 


\begin{tabular}{|c|c|c|c|c|c|c|}
\hline Ids & Fluorochromes & Clones & Isotypes & Suppliers & References & $\boldsymbol{\mu L}$ per $\mathbf{1 0}^{\mathbf{6}}$ cells \\
\hline CD45 & PerCP & D058-1283 & IgG1-Mouse & BD & 558411 & 1,5 \\
\hline CD3 & V500 & SP34-2 & IgG1-Mouse & BD & 560770 & 1 \\
\hline NKG2A & PE & Z199 & IgG1-Mouse & Beckman Coulter & IM3291U & 2 \\
\hline CXCR5 & FITC & MU5UBEE & IgG1-Mouse & ebioscience & 15566616 & 5 \\
\hline CD20 & AlexaFluor 700 & 2 H7 & IgG2-Mouse & BD & 560631 & 3 \\
\hline CD27 & PE-Cy7 & M-T271 & IgG1-Mouse & BD & 560609 & 2 \\
\hline IgD & ECD & Polyclonal & IgG-Goat & Southern Biotech & $2030-07$ & 2 \\
\hline IgG & FITC & G18-145 & IgG1-Mouse & BD & 560952 & 2 \\
\hline IgM & PE-Cy5 & G20-127 & IgG1-Mouse & BD & 551079 & 1,5 \\
\hline IgA & APC & Peptide M & NA & Mouquet's lab & $10.1016 / j . j i m .2020 .112833$ & 3 \\
\hline gp140 & Streptavidin-PE & NA & Fisher Scientific & ThermoFisher & $12-4317-87$ & 1 \\
\hline
\end{tabular}

NA: not applicable

Supplementary Table 2: Antibodies used for flow cytometry staining. Table showing antibodies used to gating immunoglobulin, SIV specificity (gp140) on memory B cells and CXCR5 on NK cells in different tissues from both species (AGM, MAC).

\begin{tabular}{|c|c|c|c|c|c|}
\hline Ids & Fluorochromes & Clones & Isotypes & Suppliers & References \\
\hline Anti-NKG2A & NA & Polyclonal & IgG-Rabbit & Abcam & ab170844 \\
\hline Anti-IgA & NA & IgA5-3B & IgG-Mouse & Abcam & ab53270 \\
\hline Nucleus & DAPI & NA & NA & Sigma & D9542-10MG \\
\hline Anti-Mouse IgG H\&L & AlexaFluor 568 & Polyclonal & IgG-Donkey & Abcam & ab175472 \\
\hline Anti-Rabbit IgG H\&L & AlexaFluor 488 & Polyclonal & IgG-Donkey & Abcam & ab150061 \\
\hline
\end{tabular}

Supplementary Table 3: Antibodies used for microscope staining. Table showing antibodies used to identify NK cells and IgA+ cells in different tissues from both species (AGM, $\mathrm{MAC})$. 